University of Redlands

\title{
Identification of Optimal Timber Harvest Locations
}

\author{
A Major Individual Project submitted in partial satisfaction of the requirements \\ for the degree of Master of Science in Geographic Information Systems \\ by \\ Peter Lecourt \\ Fang Ren, Ph.D., Committee Chair \\ Nader Afzalan, Ph.D.
}

August 2016 
Identification of Optimal Timber Harvest Locations

Copyright (C) 2016

by

Peter Lecourt 
The report of Peter Lecourt is approved.
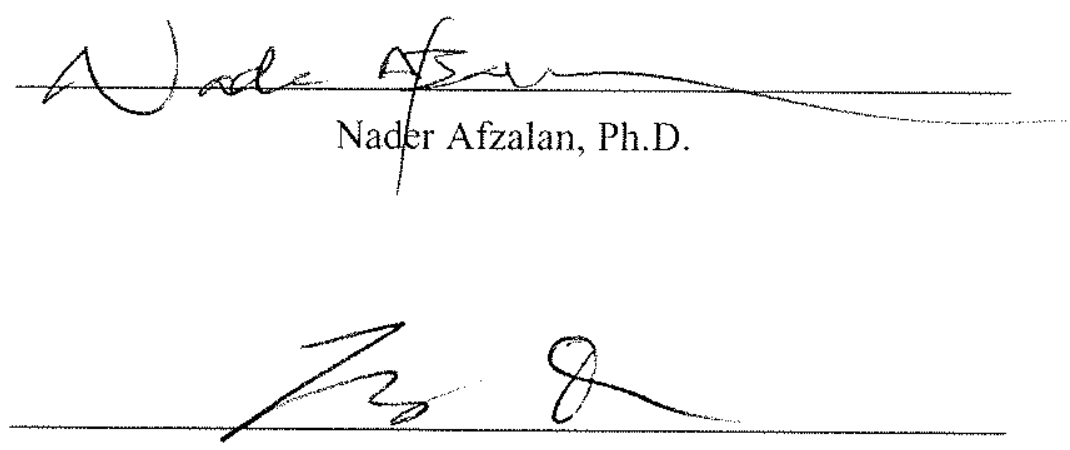

Fang Ren, Ph.D., Committee Chair

August 2016 



\section{Acknowledgements}

I would like to thank Nathan Strout, director of the UR Center for Spatial Studies, for his help with the extensive Python scripting necessary for this project. I would also like to thank my advisor, Dr. Fang Ren, for all of her help throughout the process of this project. Many thanks to the entire faculty and staff of the UR MS GIS program for their contributions to a great year studying GIS. 



\author{
Abstract \\ Identification of Optimal Timber Harvest Locations \\ by \\ Peter Lecourt
}

\begin{abstract}
More than a century of fire suppression and mismanagement have severely affected the forest and fire ecology of the Blue Mountains of Oregon. To clear these forests and raise funds for restoration, the USDA Forest Service would like to conduct timber harvests of the unnaturally abundant tree species from these forests. Harvestable areas must be compliant with the Oregon Department of Forestry's Forest Practice Rules. Once harvestable areas are identified, travel times from these areas to nearby timber mills must be generated. A tool was created with python scripting in ArcGIS to streamline this analysis. Of the 1617 square miles of the study area, 1391 square miles were found to be compliant with the Oregon Department of Forestry rules. Travel times from these compliant areas to 32 nearby timbers mills were generated with a mean travel time of 214 minutes, low travel time of less than one minute, and a high travel time of 443 minutes. The tool created by this project will be used for analysis of other forested areas in the future.
\end{abstract}





\section{Table of Contents}

Chapter 1 - Introduction ................................................................................................ 1

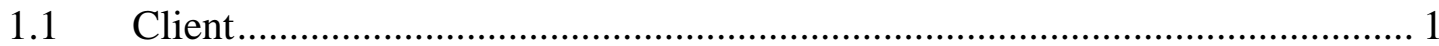

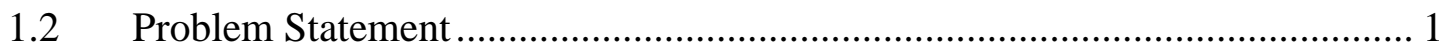

$1.3 \quad$ Proposed Solution ............................................................................... 1

1.3.1 Goals and Objectives ........................................................................... 2

1.3.2 Scope

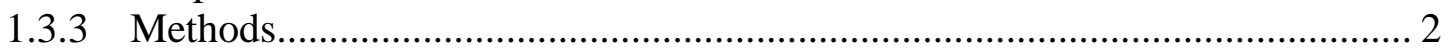

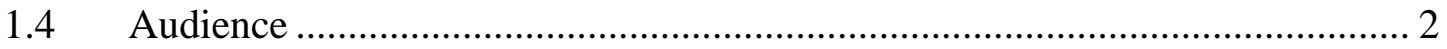

1.5 Overview of the Rest of this Report …………………….............................. 3

Chapter 2 - Background and Literature Review ................................................................ 5

2.1 Oregon Department of Forestry Timber Harvest Area Parameters ................... 5

2.2 Calculation of Travel Times of Logging ........................................................ 6

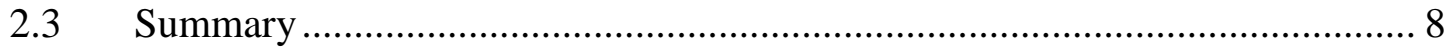

Chapter 3 - Systems Analysis and Design..........................................................9

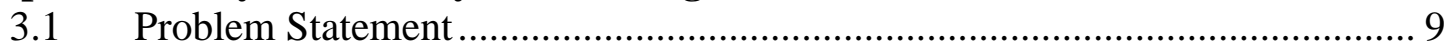

$3.2 \quad$ Requirements Analysis ……………………….................................. 9

3.2.1 Functional Requirements ...................................................................... 9

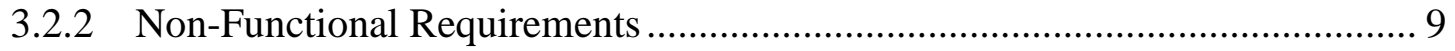

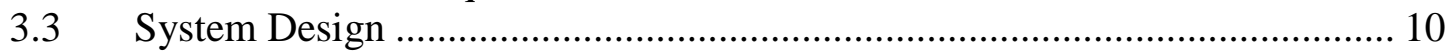

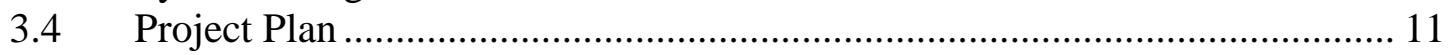

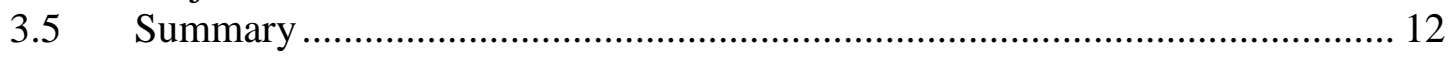

Chapter 4 - Database Design.................................................................................................... 13

4.1 Conceptual Data Model ................................................................... 13

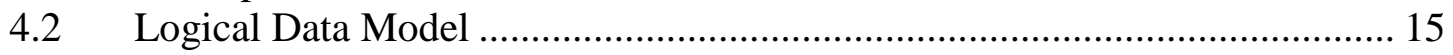

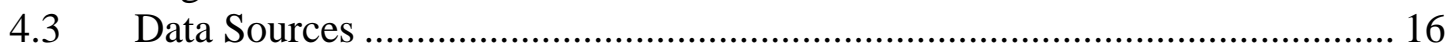

4.4 Data Scrubbing and Loading ……………................................................ 17

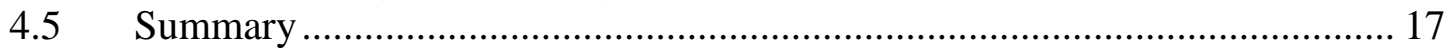

Chapter 5 - Implementation................................................................................................ 19

5.1 Generation of ODF Compliant Timberlands .............................................. 19

5.2 Generation of Travel Times ..................................................................... 22

5.3 Creation of the Optimal Timberlands with Travel Time Tool........................ 25

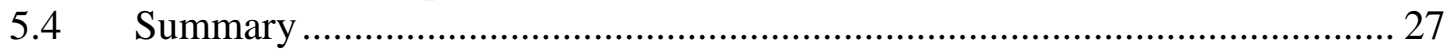

Chapter 6 - Results and Analysis...................................................................................... 29

6.1 ODF Compliant Timberlands .................................................................. 29

6.2 Travel Times from Timberlands to Mills...................................................... 30

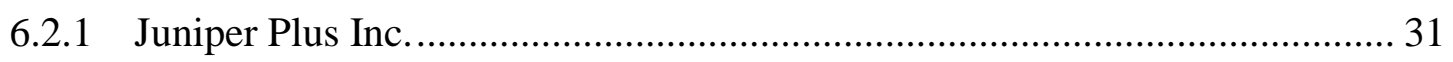

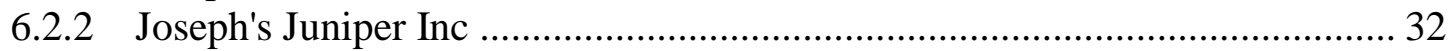

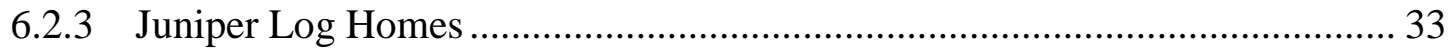

6.3 Travel Time Analysis Methodology Testing ……………………………....... 34

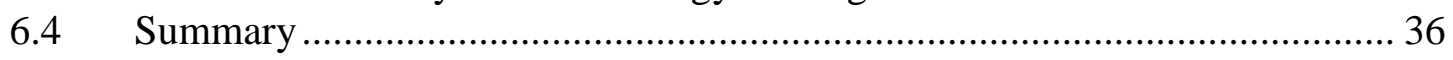


Chapter 7 - Conclusions and Future Work .......................................................... 37

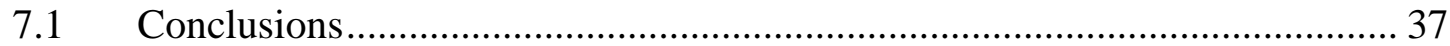

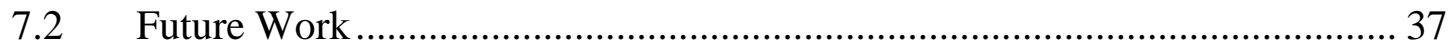

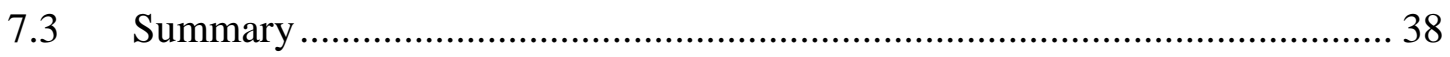

Works Cited .................................................................................................................. 39

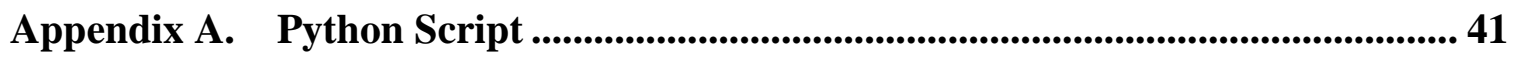




\section{Table of Figures}

Figure 3-1: System Design.................................................................................... 10

Figure 4-1: System Conceptual Data Model ...................................................... 13

Figure 4-2: $\quad$ Optimal Timberlands Conceptual Data Model ..................................... 14

Figure 4-3: Logical Data Model ....................................................................... 15

Figure 5-1: Generation of ODF Compliant Timberlands ................................... 19

Figure 5-2: Physiographic Analysis Workflow ............................................ 21

Figure 5-3: Generation of Travel Times .......................................................23

Figure 5-4: Points Generated for Timberlands ..................................................24

Figure 5-5: Optimal Timberlands with Travel Time Tool Interface ..................26

Figure 6-1: ODF Compliant Timberlands and Non-Harvestable Areas ................29

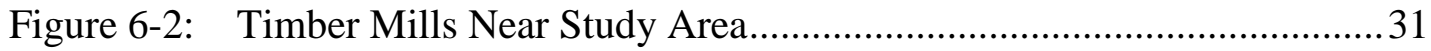

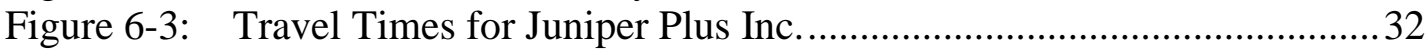

Figure 6-4: Travel Times for Joseph's Juniper Inc ........................................... 33

Figure 6-5: Travel Times for Juniper Log Homes .............................................. 34 



\section{List of Tables}

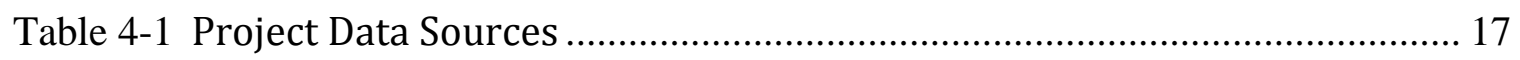

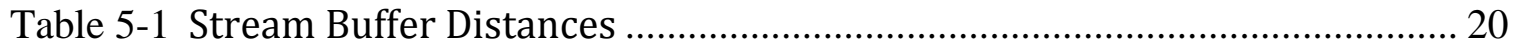

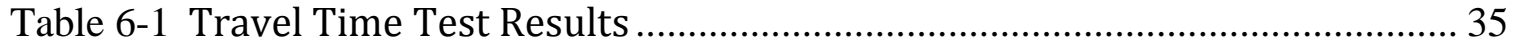

Table 6-2 Execution Time per Individual Timber Mill........................................... 35 



\section{List of Acronyms and Definitions}

$\begin{array}{ll}\text { DEM } & \text { Digital Elevation Model } \\ \text { GIS } & \text { Geographic Information Systems } \\ \text { ODF } & \text { Oregon Department of Forestry } \\ \text { USDA } & \text { United States Department of Agriculture } \\ \text { USGS } & \text { United States Geological Survey }\end{array}$





\section{Chapter 1 - Introduction}

As the dominant species on the planet, human beings have a responsibility to be good stewards of the environment. Forests represent a keystone component of our planetary ecosystem, and they are being unsustainably degraded by anthropomorphic activities. Due to such activities, the forests of the Blue Mountains of Oregon are in need of ecological restoration. This project sought to identify the best areas of the Blue Mountains which could be harvested for timber to raise funds for restoration.

Chapter 1 is organized as follows. Section 1.1 discusses the project client. Section 1.2 defines the problem statement. Section 1.3 offers the proposed solution. Section 1.4 describes the project audience, and Section 1.5 gives an overview of the rest of the report.

\subsection{Client}

The client of this project was the United States Department of Agriculture (USDA) Forest Service, which owns the land being analyzed in this project: the Blue Mountains. The Forest Service needed to determine which lands in the study area can be cost effectively harvested for timber. The technical point of contact for this project was Kevin Vogler, faculty research assistant in Oregon State University's College of Forestry. Vogler was responsible for providing some of the project data, for communicating the scope of this project, and for helping to guide the project workflow throughout the process.

\subsection{Problem Statement}

Due to a century of improper management, the forest ecology in Oregon's Blue Mountains has been severely disrupted. The objective of current management practice has shifted from timber production to recovery of the natural ecology of the area. However, due to decreasing budgets and increasing spending on wildfires, the Forest Service does not have enough money to spend on proper management and restoration of these forests.

To raise funds for restoration, the Forest Service plans to extract timber from areas that have grown too dense due to alterations of the natural forest structure. Planning a timber harvest is a complex process, and a number of factors must be carefully considered in order to find areas that can be harvested in both an ecologically and economically sound fashion. The challenge that the client faced was twofold: identifying which areas in the Blue Mountains met the harvesting criteria of the Oregon Department of Forestry's (ODF) Forest Practice Rules; and finding vehicular travel time from these harvestable areas to nearby timber mills.

\subsection{Proposed Solution}

The proposed solution to the client's problem was to develop a custom GIS tool that identifies which areas of a forest are compliant with the ODF Forest Practice Rules, and then provides a travel time from those areas to nearby timber mills. 


\subsubsection{Goals and Objectives}

Restoration of forested lands is an expensive, labor intensive process. The goal of this project was to determine which areas in the Blue Mountains could be harvested for timber to produce the most funds for ecological restoration. The project objectives were to identify which areas of the Blue Mountains could be harvested to meet ODF standards, and to find the travel times from the harvestable areas to nearby timber mills.

\subsubsection{Scope}

The study area for this project covered 1,617 square miles of mountainous forested terrain in eastern Oregon. Factors and data that were considered when planning timber harvesting on the study area included wildlife (location of sensitive species), geologic composition (boundaries of geologic units), slope (digital elevation model, or DEM), and proximity to streams (stream network), lakes (location of water bodies), roads (road network) and timber mills (mill locations). A custom-built timberland area tool that outputs optimal timber harvest locations within the study area was the main deliverable for this project. The output also provides travel time from specific timber mills to the optimal harvesting locations.

\subsubsection{Methods}

The custom timberland area tool created for this project was constructed using Python scripting. To create a basic structure of the Python script, geoprocessing tools were input into the ArcGIS ModelBuilder, and this model was exported to a Python script. The script was then modified to match the necessary specifications of this project. Some tool parameters were pre-set in the timberland area tool, and others had to be specified by the end user.

Project data came from a variety of sources, including the project client, Esri, the Oregon Department of Forestry, the USDA Forest Service, and the United States Geological Survey. Most project data received were ready for input into the timberland area tool without significant changes. The main dataset requiring significant alteration was the stream data, which required the addition of a new field showing the stream class for each record. This field was calculated using information from other fields in the stream data.

\subsection{Audience}

This report was written for forestry professionals. Geographic Information Systems (GIS) are widely used in forestry, and many professionals in the field have a fundamental working knowledge of GIS. Readers will be familiar with many of the basic tools and operations discussed in this report. However, the more advanced concepts, such as python scripting, may be unfamiliar to the audience. These concepts will need to be more thoroughly explained to help the intended audience have a more complete understanding of the steps carried out during the execution of this project. 


\subsection{Overview of the Rest of this Report}

The rest of this report is organized as follows: Chapter Two covers the background research at the base of the project, including a discussion of the information that guided the execution of this project. Chapter Three illustrates the design and creation of the timberland area tool. Chapter Four is about the collection and storage of data utilized in this project. Chapter Five shows how the timberland area tool was implemented to provide the information that this project sought to discover. Chapter Six examines the results and analysis of the project. Chapter Seven discusses the conclusions of the project, and future work that could be built upon it. 



\section{Chapter 2 - Background and Literature Review}

The harvesting of timber must be carefully planned and executed. A number of environmental factors must be considered to ensure that harvesting is sustainable and does minimal damage to the environment. Locations of timber harvests must also be considered from an economic standpoint to ensure they are close enough to timber processing facilities to allow for minimal transportation expenses during the harvest.

A literature review was conducted to gain a better understanding of what kinds of environmental factors must be considered when planning timber harvests, and how those factors affect both the abiotic and biotic features of an ecosystem. Literature on the use of GIS to plan logging truck routes was also examined. The following chapter summarizes the research on these two topics.

\subsection{Oregon Department of Forestry Timber Harvest Area Parameters}

The Oregon Department of Forestry has specific guidelines on how timber harvests are to be conducted in Oregon, which are provided in a 94-page document entitled the Forest Practice Rulebook. The rules are based on a variety of scientific information demonstrating how timber harvesting affects the environment in a number of ways. These rules dictate how timber harvesting is to be conducted in consideration of slope and geology, aquatic features such as streams and lakes, and wildlife.

Rules of slope and geology are meant to "prevent landslides, which cause sedimentation of streams" (ODF, 2014). Studies examining how logging impacts stream sedimentation levels were first conducted in the late 1970s by William Dietrich (1978), who found that the creation and use of unpaved forest roads lead to increased levels of sedimentation in nearby rivers and streams. This led to more research on the impacts of forestry practices on stream sedimentation. The greatest cause of stream sedimentation was found to be from landslides caused by forestry practices (Jordan, 2006). Landslides occur with increased frequency on steep terrain following timber harvesting activity, especially following the practice of clear cutting (Imaizumi, Sidle, \& Kamei, 2008). This is primarily due to the decomposing of tree roots post-harvest, which are responsible for providing a solid framework for steep soils. However, environmental factors affecting the rate of root decay are poorly understood. Imaizumi, Sidle, and Kamei (2008) also found that the effects of forest harvesting on slope stability is strongly affected by geology and soil type. Some soil types will erode more easily on shallower slopes. Decomposed granitic soils and easily erodible soils must be treated differently than other soil types when harvesting timber on uneven terrain, as they are more likely to cause landslides (ODF, 2014).

Increased stream and lake sedimentation from landslides, as well as from unpaved forest roads and timber harvesting near surface water, has a number of negative impacts. From the anthropomorphic view, sedimentation is bad because many people in rural areas depend on streams and lakes for their home water use, and this water is often minimally treated before use. Many fish species are also negatively impacted by the increase of aquatic sedimentation (Ramezani, Rennebeck, Closs, \& Matthael, 2014). Fish eggs laid in streams require a certain oxygen concentration, which is maintained by a constant flow of water over the eggs. This flow is blocked as sediments accumulate on the eggs (Grieg, 
Sear, \& Carling, 2005). Sediment addition also reduces the total taxonomic richness of benthic invertebrates, which are a major source of food for fish (Ramezani, Rennebeck, Closs, \& Matthael, 2014). Increased sedimentation raises water temperatures

(Kreutzweiser \& Holmes, 2009), which leads to a decrease in dissolved oxygen, affecting survival of adult fish as well as of eggs (Ling et al., 2016). Water temperature

significantly affects a number of biological and chemical processes in aquatic ecosystems (Caissie, 2006), so it is important for logging activities to have minimal impact on stream temperature. This can be achieved by reducing sedimentation, as well by increasing the amount of canopy cover near streams which provides shade. Riparian buffers, which are areas near streams and lakes that are left unharvested, provide this shade. They also provide shoreline stability, water quality protection, and wildlife habitat (Kreutzweiser \& Holmes, 2009). The Forest Practice Rulebook specifies rules guiding riparian buffers in logging sites with "the overall goal...to provide resources protection during operations adjacent to and within streams, lakes, wetlands and riparian management areas so that, while continuing to grow and harvest trees, the protection goals for fish, wildlife, and water quality are met" (ODF, 2014).

The Forest Practice Rulebook also specifies protective buffers of forested area around the nests of three species of wildlife: the Great Blue Heron, the Bald Eagle, and the Spotted Owl. In 1990, the Northern Spotted Owl was formally declared to be a threatened species, due primarily to heavy logging (Egan, 1990). This led to a greater awareness of the effects of logging on tree-nesting avian species. Saber Ghasemi (2015) found that these species are highly sensitive to alterations in their environment, and that they display a wide range of susceptibilities to habitat modification. He also found that generalist species, which are able to feed on a wider variety of foods, are less affected by logging than specialists. An upset of the environment, such as a timber harvest, can easily eliminate the food sources of specialist species. More protection must be given to such species. Models which consider planned logging activities are used to predict the responses of individual species to the logging, and greater protections can then be given to those species (Tobias, 2015). Species that are heavily impacted by logging receive protection in the ODF Forest Practice Rulebook. Thus, these species, including the Bald Eagle, the Great Blue Heron, and the Spotted Owl, should be considered when planning logging activities.

\subsection{Calculation of Travel Times for Logging}

Timber companies have long faced the problem of finding optimal routes to transfer timber from logging sites to timber mills. The routing of logging trucks has traditionally been a manual process performed by transport planners (Anderson, Flisberg, Liden, \& Ronnqvist, 2008). The goal of such planning is to decrease the cost of transportation per mile and increase revenue per mile (Delvin, McDonnell, \& Ward, 2008). Routing used to depend on traditional Euclidian distance measurement, but has been replaced with more useful measurements, such as vehicular travel distance or travel time (Delamater, Messina, Shortridge, \& Grady, 2012). Modern GIS systems are well suited to perform route planning (Anderson, Flisberg, Liden, \& Ronnqvist, 2008). Using GIS to find the best route from a source to a destination can be done with two primary methodologies: vector-based techniques which route along a discrete network, and raster-based techniques which can route across a continuous surface (Gonclaves, 2010). 
Dennis Dean (1997) described raster based techniques in the following way: raster cost-distance techniques work by assigning each raster cell a value associated with the cost of traversing that cell. The neighbors of a cell are examined and the neighbor containing the lowest cost value is identified. The process then continues onto this lowest cost neighbor, and repeats until the destination has been reached. The determination of which cells are neighbors can have a considerable effect on this process. Common patterns to determine neighbors are rook and queen contiguity.

Raster-based techniques have a number of strengths. Cells that represent areas with roads have low values, and off-road areas have higher values. Since raster surfaces are continuous, routes can be drawn to areas that are not in contact with any kind of road (Gonclaves, 2010), although the routes will remain on a road as long as possible. Rasterbased techniques also allow for a large number of factors to be considered when planning a route, including distance, speed limit, road owner, road width, road surface, and slope (Anderson, Flisberg, Liden, \& Ronnqvist, 2008). These factors can easily be manipulated, according to Harri Anikainen (2013), changing their effects on the cost associated with crossing each cell. He also notes that raster techniques tend to be less computationally demanding than vector based techniques.

Raster-based techniques also have a number of weaknesses. When using these techniques integrated with a discrete road network, the blocking affect introduced by working with raster data creates a jagged appearance of the road network, and overestimation of the travel cost along a link often occurs (Choi, Um, \& Park, 2013). As a result, over-estimation of distance is common when working with raster data, and routes may appear unrealistic when displayed on a map (Antikainen, 2013). Antikainen also found that paths generated from the node-link structure of raster techniques are often subject to distortions, though this can be improved by using neighborhoods larger than the typical $3 \times 3$ window used by many analysis techniques. A final issue is that there is no natural way to identify points where separate, unconnected routes cross when using raster techniques. An example is when an overpass crosses a highway. Raster techniques lack methods to develop topological rules on analyzed data, and may allow a vehicle to turn from a main route onto an overpass even though the two route segments are not connected in the real world.

On the other hand, discrete vector networks do allow for the establishment of topological rules on data included in analysis. Overpasses can easily be marked to be at a higher elevation than highways, and the software will not create a route that turns from a highway directly onto an overpass (Choi, Um, \& Park, 2013). Vector data also allow for the modeling of turn delays. When turns are encountered, routes can be forced to decrease in speed, allowing for more accurate modeling of travel time (Delamater, Messina, Shortridge, \& Grady, 2012). Another strength of vector techniques is that they not subject to the same blocking involved with raster data, resulting in more precise travel estimates compared with raster techniques (Antikainen, 2013).

Vector techniques have weaknesses as well. First, they require a network dataset in order to function. The creation of an accurate, comprehensive network dataset can be very time consuming (Loreno, 2014). Second, vector techniques require origins and destinations to be in contact with the network dataset, which can create problems when calculating travel times to remote areas that are not in contact with a road network (Gonclaves, 2010). Third, while vector-based techniques do have the advantage of 
providing exact paths, these techniques come with much higher computational expense (Antikainen, 2013).

Both vector- and raster-based routing techniques have strengths and weaknesses. To say that one is superior to the other is inaccurate. This is due in part to the fact that true reference data to which the comparison of results could be made does not exist. There are situations in which either technique would work. Due to the continuous nature of raster data, raster techniques are better when identifying travel paths to areas that are not in contact with a road network. Vector techniques tend to be superior if all destinations lie on or near a road network.

\subsection{Summary}

The process of timber harvesting can have a great impact on the forests being harvested. Landscapes physiography can be altered, water can be degraded, and wildlife species can be significantly affected. In order to prevent such impacts, Oregon has specific guidelines that outline requirements of how timber must be harvested within the state. These guidelines are built upon scientific research, and will be followed throughout the execution of this project.

Choosing sites for timber extraction is based not only on environmental concerns, but on economic concerns, as well. In order for a timber harvest to produce adequate profits, transportation planning is crucial. Identifying the travel time for logging trucks to travel between a mill and timber harvest sites helps forestry professionals determine which are the optimal sites to harvest. GIS is well suited to aid in this process.

GIS Spatial Analyst tools will be used to identify which areas of a forest are compliant with the ODF Forest Practice Rules. Once the compliant areas have been identified, Network Analyst tools will be used to calculate travel times from timber mills to the ODF compliant areas. Vector-based Network Analyst tools were chosen over raster-based tools since logging trucks transporting timber only travel on a road network. These analyses and other system requirements are outlined in the next chapter. 


\section{Chapter 3 - Systems Analysis and Design}

In order to ensure that client needs will be met, project requirements must be identified and implemented into the system design. The purpose of this chapter is to outline the system requirements, design, and implementation. The chapter starts with a statement of the client's problem. Next, project requirements are examined. System design follows, which leads into the project plan.

\subsection{Problem Statement}

In order to raise funds for restoration of forests in the Blue Mountains of Oregon, the USDA Forest Service would like to extract non-naturally thriving timber species from these forests. This project sought to aid the Forest Service in that process. The problem addressed by this project was twofold. First, the need to identify which areas of a forest are compliant with the ODF Forest Practice Rules. Second, calculating travel time from nearby timber mills to these ODF compliant timberlands.

\subsection{Requirements Analysis}

The deliverable of this project was a Python script tool that must achieve two outcomes: identification of areas within a forest that are compliant with the ODF Forest Practice Rules, and calculation of travel times from nearby timber mills to these ODF compliant timberlands. In order to ensure that these outcomes were achieved, a requirements analysis of the project was conducted. These included both functional and non-functional requirements, which are addressed below.

\subsubsection{Functional Requirements}

Functional requirements define specific functionalities that a system is meant to accomplish. They can be inputs, processes, and outputs. The system has four functional requirements: the system must allow the user to supply the various data and parameters necessary to run the analysis; the system needs to identify the areas of a user-specified forest that are compliant with the ODF Forest Practice Rules; the system must calculate vehicular travel times to get from user-specified timber mills to the ODF compliant forested timberlands; and the system must output one feature class for each timber mill supplied by the user which shows the travel times from a specific mill to the ODF compliant timberlands.

\subsubsection{Non-Functional Requirements}

Non-functional requirements support functional requirements, and specify criteria of the design and implementation of the end product. The system has six non-functional requirements. First, the system shall require specific data necessary for the system analysis. Second, the Oregon Optimal Timberlands Tool must be run on ArcGIS version 10.0 or higher, and requires both the Spatial Analyst and Network Analyst extensions. Third, the system must be run on a computer running the Windows operating system, 
version XP or more recent. Fourth, the computer's minimum hardware requirements are a 2.2 $\mathrm{GHz}$ dual core processor, $2 \mathrm{~GB}$ of RAM, with $2.45 \mathrm{~GB}$ of free disk space. Fifth, the system must have a user interface that is flexible to account for different possible site conditions. For instance, the system generates a 300-foot buffer for Great Blue Heron nests. If no such nests exist on the site, the system must be able to run despite lacking this input dataset. Sixth, the system will be provided with a user tutorial, that describes the analysis and the data required for the system to function.

\subsection{System Design}

After establishing project requirements, plans for the system design were constructed. Figure 3-1 depicts the system design.

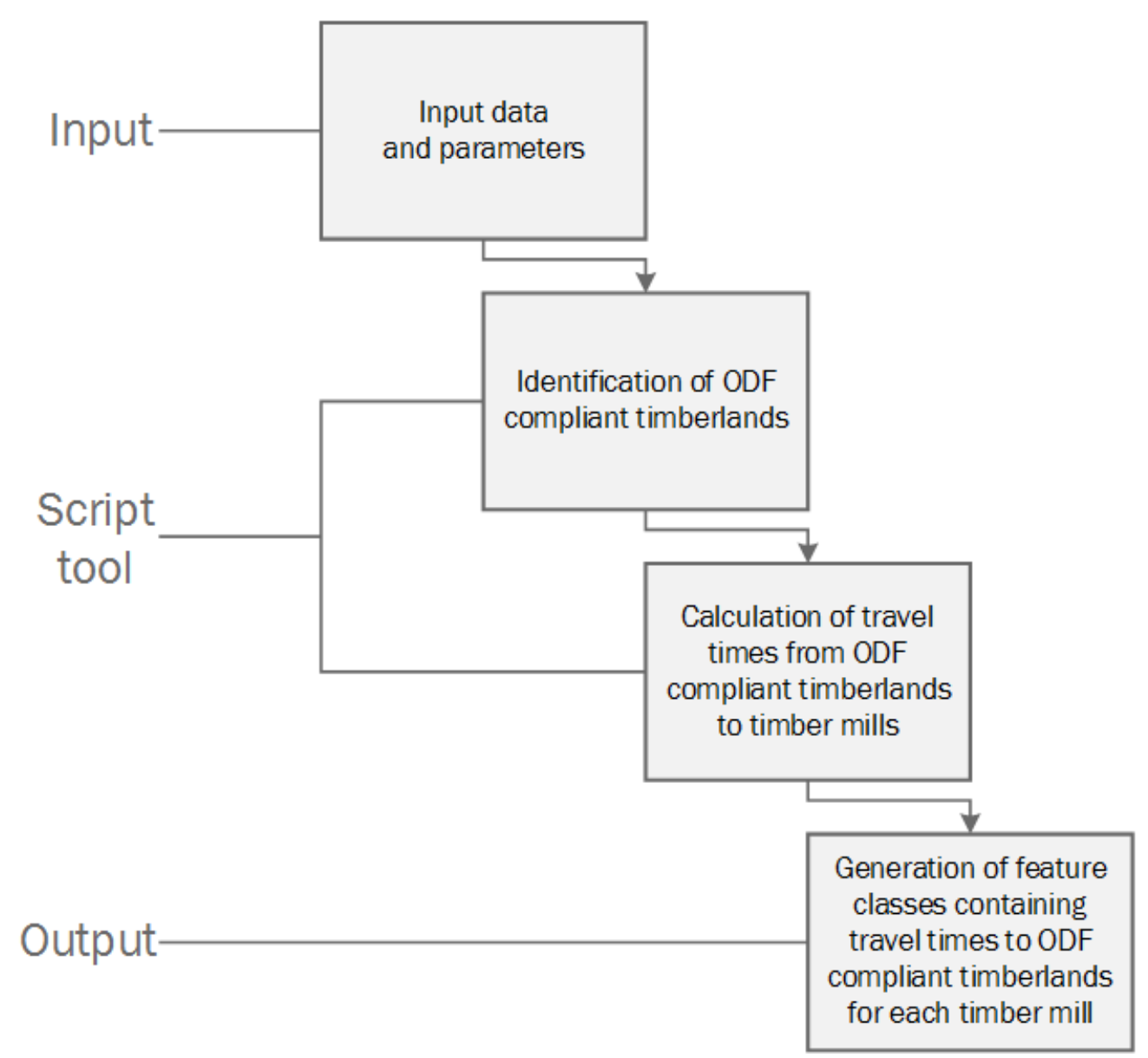

\section{Figure 3-1 System Design}

The system consists of a tool that can take specific input data, find ODF compliant forested areas, generate travel times from ODF compliant timberlands to nearby timber mills, and output feature classes containing data produced by the analysis. Python scripting is well suited to automate this work flow and generating such a tool, so the core component of the system is a Python script. This script prompts users to input the various 
required datasets, and contains all tools necessary for the analysis, as well as the ODF timber harvesting parameters which guide tool execution. A custom Python script tool named Optimal Timberlands Tool with Travel Time was constructed from the script, which has a user-friendly interface that simplifies data and parameter input into the script.

The script has two main workflows, both encompassing a number of geoprocessing tools. The first identifies all areas of the input forestlands that are not compliant with the ODF timber harvesting parameters and removes these areas from the forestlands, giving an output which shows all areas of the forestlands that are ODF compliant. The second workflow calculates travel times from the user-specified timber mills to the areas comprising the ODF compliant forestlands. The script produces one output for each mill input into the system, which shows the travel times generated for that specific mill.

\subsection{Project Plan}

A project plan is like a roadmap. It lays out the plans for how a project will be executed from start to finish. Project plans are typically divided up into phases, and each phase consists of a number of tasks. Five phases were identified for this project.

Phase One of the project was project definition. In this phase, the project was selected. Discussions with the client then helped to identify the project definition, scope, and possible solutions. Phase Two was project planning. Continued contact with the client helped to identify what resources would be required to implement project execution, possible project workflows to address project scope, and the feasibility of the project. Research was conducted to identify how others have solved similar problems. Phase Three was project execution. All project data were collected and organized and models were built to plan project workflows. Successful models were turned into Python scripts, and scripts were converted into a Python script tool with a user interface. Phase Four was project implementation and testing. Project data were run through the tool, and the outputs were examined and tested. Phase Five was project closing. The Python script tool, project data, and user documentation were delivered to the client, together with technical support.

During the course of project execution, the scope of the project and the project timeline changed significantly. The original scope of the project was the identification of travel times from timber mills in Oregon, Washington, and Idaho to forested areas in the Blue Mountains of Oregon. However, to better locate the appropriate timber sites, the scope was expanded to involve finding forested areas that are compliant with the ODF

Forest Practice Rules. This task involved the collection of a variety of data representing factors that must be considered when harvesting timber in Oregon, as well as the combination of geoprocessing tools to run the required analysis on those data.

Many of the forested lands in the project study area are not in contact with a road network. The original plan for routing analysis involved incorporation of raster techniques that are able to identify travel times across a continuous surface. However, when timber is extracted in remote areas, heavy logging equipment is used to transport timber to logging trucks, which remain on the road network. Once this point had been clarified, workflows involving raster-based routing techniques were removed from the project plan. A workflow to transform the 46,000 polygons of the study area into point features that could be efficiently used as destinations in the Network Analyst Origin Destinations Cost Matrix was planned instead. 
Changes will occur during any project, and realistic estimation of the project timeline is a challenging task. Tasks often take more time or resources than originally expected, or new tasks may be added which increase the project scope, affecting the project timeline. However, this can sometimes be offset by other tasks that either take less time than expected, or that can be eliminated. The process of project planning and execution is complicated by unexpected factors that arise. This requires the project manager to be flexible, working with complications as they arise.

\subsection{Summary}

This project identifies a set of forested areas compliant with ODF standards, and calculates travel times from timber mills to the compliant areas. A set of functional and nonfunctional requirements had to be identified and created to successfully carry out the

project. The system designed for the project combined multiple geoprocessing tools in a Python script which allows users to enter data and parameters to produce the final result. The project scope and requirements changed during the course of the project, which required flexibility in making alterations to the project plan. 


\section{Chapter 4 - Database Design}

Geographic data lie at the heart of any GIS project. For optimal use of these data, proper organization within a database is required. The Esri geodatabase was utilized to store project data. All project data except the road network were incorporated into the geodatabase prior to analysis. Feature classes and raster datasets were easily imported, but shapefiles required conversion to these formats before integration into the geodatabase.

This chapter discusses the design and implementation of the project geodatabase. Section 4.1 looks at the conceptual data model, which is an outline of the project database. Section 4.2 discusses the logical data model, which brings the abstract conceptual database into the implementation database model. Section 4.3 lists the project data sources. Section 4.4 describes the data scrubbing and data loading used to prepare data for analysis.

\subsection{Conceptual Data Model}

A conceptual data model is a high-level conceptual diagram of a database that captures main entities and the relationships between the entities required to solve a problem. Data entities are linked together by different kinds of relationships which help reach the eventual project solution and output. This provides a template of how data will be stored in a database, and the workflow required to create the project outcome. It is often useful to use Unified Modeling Language (UML) diagrams to illustrate conceptual data models. Figure 4-1 is a UML diagram depicting the broad structure of the system's data model.

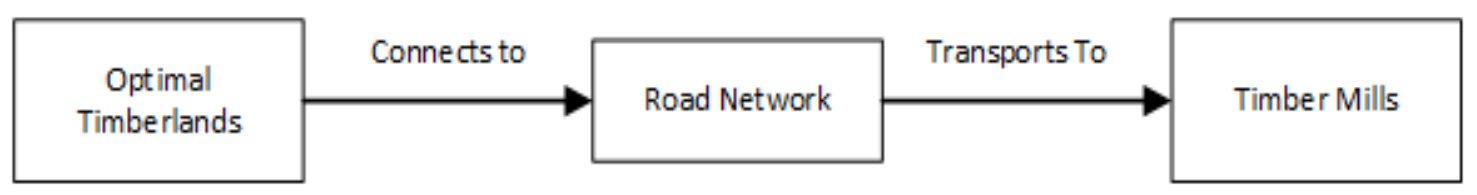

Figure 4-1 System Conceptual Data Model

This model shows how the three primary entities (timber mills, optimal timberlands, and a road network) are related. In order to identify the optimal timberlands with travel times, travel times from the optimal timberlands to the timber mills must be calculated using the road network. The road network connects the polygons of the optimal timberlands to the timber mills. The relationship between the timber mills and the optimal timberlands is many-to-many. The generation of the optimal timberlands from the input forest boundary involves a number of environmental factors which are depicted in Figure 4-2. 


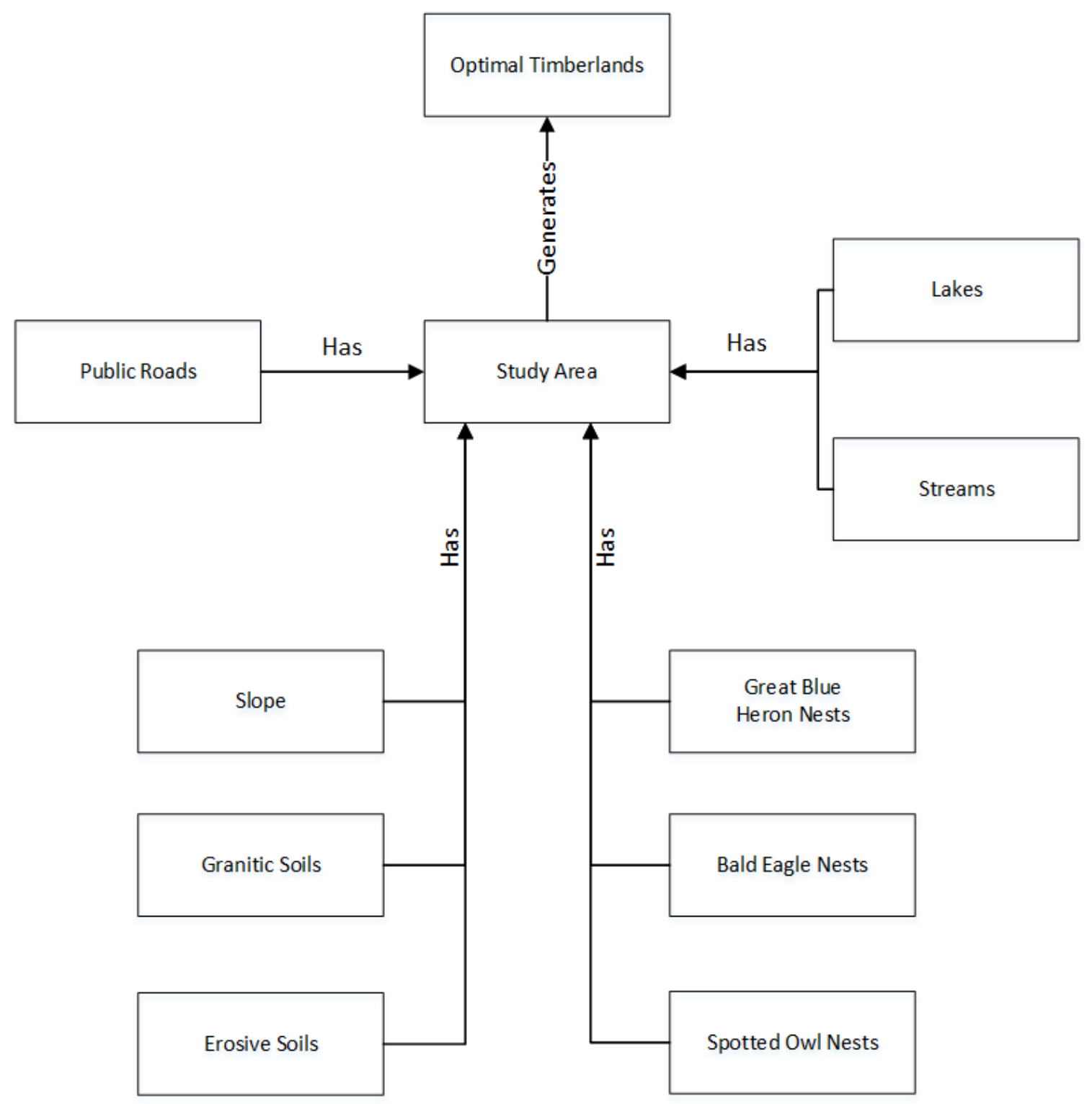

Figure 4-2 Optimal Timberlands Conceptual Data Model

This figure depicts the relationship between the optimal timberlands entity and the various entities used to generate it: the study area, public roads, slope, granitic soils, erosive soils, Great Blue Heron nests, Bald Eagle nests, Spotted Owl nests, streams, and lakes. These entities fall into four broad categories: transportation, physiography, wildlife, and aquatic features. The branching of the diagram represents the grouping of entities in these four categories. The study area contains the physical and biological features represented by the other entities below it on the UML diagram. Each of these entities contains multiple features, and the study area consists of multiple polygons. Therefore, the relationship between each of these entities and the study area is many-tomany. 


\subsection{Logical Data Model}

Once a conceptual model of the theoretical construction of a database is drawn, the physical structure of the database can start to be constructed. The logical data model transforms the conceptual design into an actual physical database. The database used in this project is an Esri File Geodatabase, which helped to organize project data and provide easy data access and management. Entities from the conceptual model are stored as feature classes and raster datasets within the geodatabases. Feature datasets, which are similar to folders within a filing cabinet, are used to group together feature classes depicting similar types of information. Figure 4-3 shows the structure of the file geodatabase created for this project.

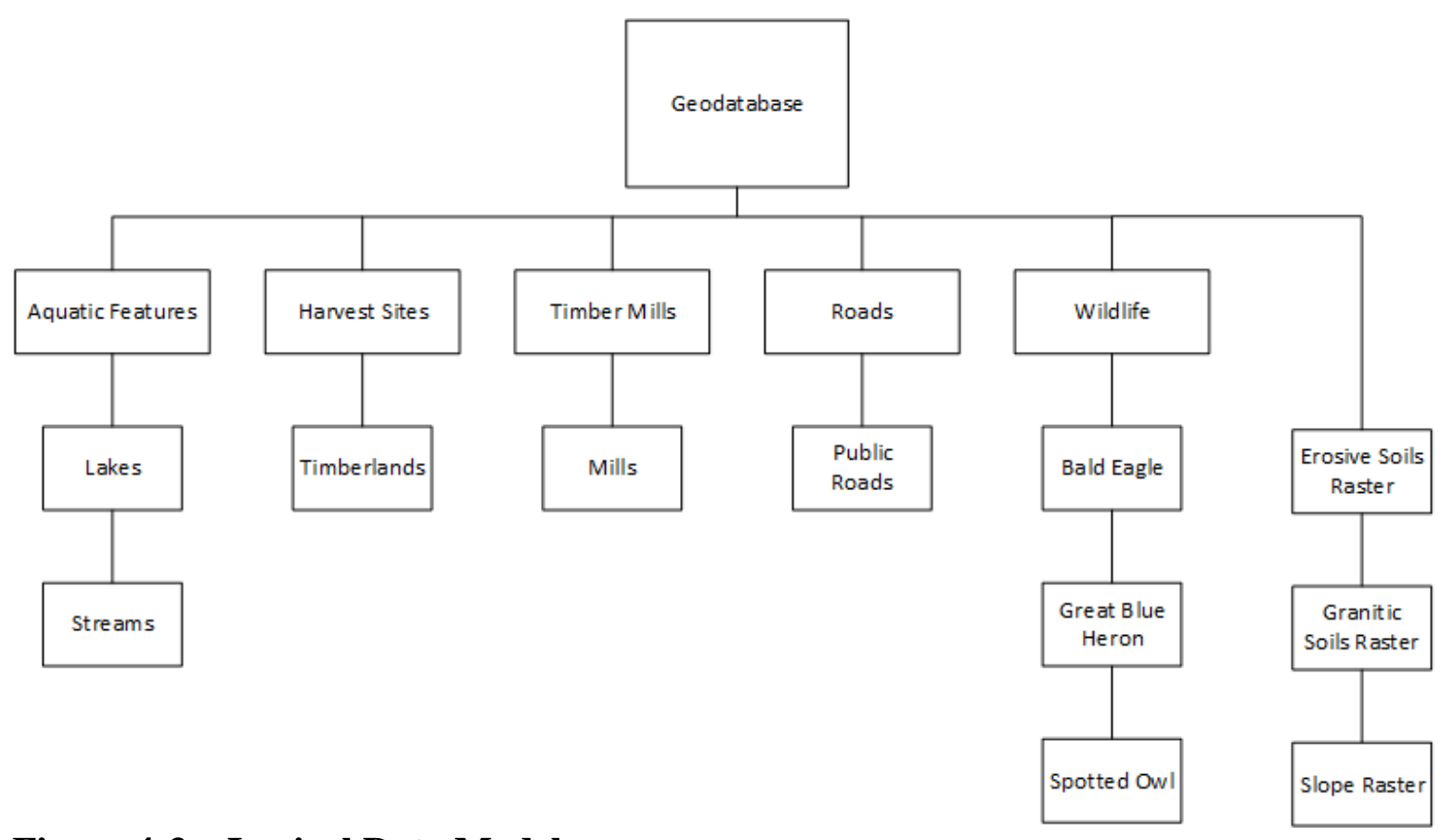

Figure 4-3 Logical Data Model

The geodatabase contains five feature datasets, which were created to provide structure and organization of information within the geodatabase. Each feature dataset contains similar features that were analyzed together during the project analysis. The first is for aquatic features, which contains the streams and lakes feature classes. Streams are linear in nature, so the streams feature class has a linear geometry. Lakes could be represented as point features for some uses, but the project analysis required lake size, so lakes are represented with polygonal geometry. The second feature dataset is for harvest sites, and contains the timberlands feature class that comprises the study area. While the area of these harvest sites is not used directly by the project analysis, harvest site shapes were irregular, and harvest size area is required for post-analysis use of the data. Therefore, harvest sites are represented with polygonal geometry. The third feature dataset is for timber mills, and contains a feature class of mills in the vicinity of the study area. Timber mills occupy discrete locations, and the size of the timber mill is not 
required by the analysis, so timber mills have point geometries. The fourth feature dataset is for public roads, and contains a feature class depicting public roads in the study area. Roads are commonly constructed with linear geometry in GIS, and this convention was followed by this project. The fifth feature dataset is wildlife, and contains feature classes for the three species of wildlife that are protected by the ODF timber harvest parameters. The feature classes represent the location of nests, which do not occupy a significant area, and are depicted with point geometries.

The geodatabase also contains three raster datasets, depicting erosive soils, granitic soils, and slope. While all of these features are physiographic, and can be grouped in a feature dataset, the feature dataset format does not allow the inclusion of raster datasets. Slope data were derived from a DEM, which is depicted in a raster data format. Raster data were required for the project's physiographic analysis, and slope was thus kept in a raster format. Erosive soil data were also obtained in a raster format, and was kept as such. The granitic soils data were originally in a vector file format. However, due to the necessity of raster data as inputs into the physiographic analysis, the granitic soils data were transformed into a raster format.

All data required for project analysis were included in the project geodatabase, except for the road network. ArcGIS did not allow the importation of that road network into the project geodatabase, so the road network was stored in a Windows file folder.

\subsection{Data Sources}

Data for this project came from a variety of sources (Table 4-4). The client provided the study area and timber mill location data. The Esri road network, entitled StreetMap North America, was provided by the University of Redlands Center for Spatial Studies. This road network dataset provided a comprehensive set of roads outside of the study area. Of the 1,617 square miles of the study area, 1,594 square miles were within one mile of at least one road segment from the StreetMap dataset, providing 98.5\% coverage of the study area at this one-mile distance. The StreetMap dataset was the source of the roads in the public roads dataset. The granitic soils data were made available by request from Michael Buren, Geotechnical Specialist at the ODF. The erosive soil data were obtained from the United States' Geological Survey (USGS). The elevation data which were used to generate slope came from the USGS as well, and is a part of the National Elevation Dataset (NED). The lakes data also came from a USGS product, entitled the National Hydrography Dataset (NHD). A subset of the NHD for Oregon was obtained from the online Oregon Spatial Data Library. Stream data were obtained from the Oregon Department of Forestry. All project datasets listed here were provided with metadata, except for the granitic soils data. This dataset was obtained by the ODF from the USGS, but without metadata.

Datasets representing the location of wildlife nests (Great Blue Heron, Bald Eagle, and Spotted Owl) were not available to the public, as locations of sensitive species' nests are kept private to help protect those species. Artificial data representing the location of nests for these species had to be created. A feature class was created in the project database to represent nests for each of these species, and 10 points for each feature class were randomly distributed across the study area. 
Table 4-4 Project Data Sources

\begin{tabular}{|l|l|}
\hline \multicolumn{1}{|c|}{ Dataset } & \multicolumn{1}{c|}{ Source } \\
\hline Project Study Area & US Forest Service \\
\hline Wildlife (G.B. Heron, B. Eagle, S. Owl) & Unavailable (artificial data created) \\
\hline Road Network / Public Roads & Esri StreetMap \\
\hline Streams & ODF \\
\hline Lakes & USGS (National Hydrography Dataset) \\
\hline Elevation & USGS (National Elevation Dataset) \\
\hline Soils & ODF \\
\hline Timber Mills & US Forest Service \\
\hline
\end{tabular}

\subsection{Data Scrubbing and Loading}

The majority of project data came from reputable sources, and the project data did not require a significant amount of data scrubbing. All project data (except the road network) had to be projected in to the World Geographic Coordinate System of 1984 to match the coordinate system of the road network dataset. Most project datasets encompassed areas that are larger than the project study area, so these datasets had to be clipped to the boundary of the project study area. These datasets were the public roads, streams, lakes, slope, and both soils data.

The slope data generated for this project was created by inputting a DEM into the ArcGIS slope tool, which is part of the Spatial Analyst toolset. Two USGS DEMs had to be combined to cover the study area, so the ArcGIS Mosaic to New Raster tool was used to combine the two DEMs.

The physiographic analysis required raster datasets. The granitic soils dataset was obtained in vector format, so the vector data had to be converted into a raster.

The three raster datasets used for this project had the same 10-meter cell size, but the cells did not perfectly align. To correct this, when the granitic soil and erosive soil data were projected to WGS 84 after projection of the slope raster, they were snapped to the slope raster using the Snap Raster option, found under processing extent in the environment settings of the project tool. Using the Reclassify tool, cells of the granitic soil raster were assigned a value of 1 in locations where soils were granitic, and all other cells were assigned a value of 0 . The same process was used to identify the location of erosive soils for the erosive soil raster.

\subsection{Summary}

Data are among the most important elements of any GIS project. Thoughtful organization and storage of data provide easy data access and management, often aiding complex workflows. This project used an Esri File Geodatabase to contain project data. Data must come from reputable sources, and be accompanied by metadata to judge their validity, accuracy, and utility to any project. If required datasets are not available, the data must be collected, or in the case if this project, created artificially. Many data require some kind 
of scrubbing and preparation to be used in analysis, and the data used in this project were not an exception. 


\section{Chapter 5 - Implementation}

Chapter Five explains the implementation of the project plan into the Optimal Timberlands with Travel Time tool. This tool was constructed from a Python script, which is composed of two main workflows. The first takes an input forest boundary, and finds areas of that forest that are compliant with the Oregon Department of Forestry's (ODF) Forest Practice Rules. The second calculates vehicular travel times from the ODF compliant timberlands to nearby timber mills.

\subsection{Generation of ODF Compliant Timberlands}

The ODF compliant timberlands analysis was implemented using the ModelBuilder application in ArcGIS. Once models of all parts of the analysis were successfully executed, the models were turned into Python scripts. ODF parameters were built into the scripts, and the scripts were then combined. The different aspects of the analysis involved consideration of the following factors: wildlife, public roads, aquatic features, and physiography. Figure 5-1 depicts the analysis workflow.

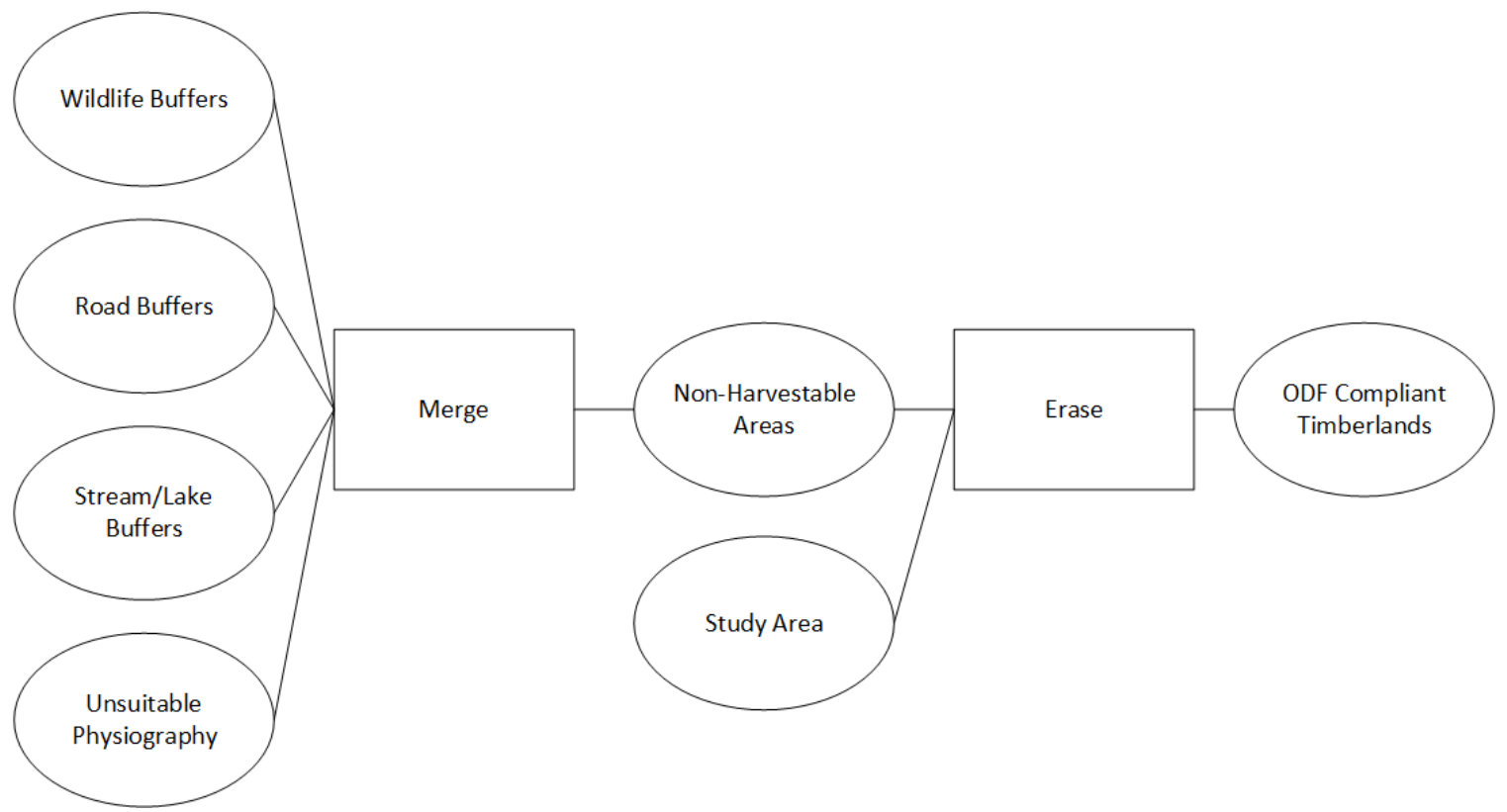

\section{Figure 5-1 Generation of ODF Compliant Timberlands}

The wildlife and public roads analysis involved creating buffers for these features using the buffer tool. Each species of wildlife is assigned a specific buffer distance, based on ODF parameters. Great Blue Heron nests are buffered 300 feet. Bald Eagle nests are buffered 330 feet. Spotted Owl nests are buffered 985 feet (70 acres). Public roads are assigned a buffer of 150 feet on either side of the road.

Aquatic features, such as streams and lakes, were also assigned buffers according to ODF standards. However, buffer distances for these features vary depending on the characteristics of each individual stream or lake. Stream buffer distance depends on the 
size of the stream, as well as the stream's classification. Stream sizes are small, medium, and large: small streams have an average annual flow of two cubic feet per second or less; medium streams have an average annual flow between two and 10 cubic feet per second; large streams have an average annual flow of 10 cubic feet per second or greater. Stream classification types are F, D, and N: streams with domestic water use are classified as type D; streams with fish, as well as streams with domestic water use that contain fish are classified as type F; streams not used as domestic water that do not contain fish are classified as type N. Different combinations of stream size and stream class receive different buffer distances (Table 5-1).

Table 5-1 Stream Buffer Distances

\begin{tabular}{|l|c|c|c|}
\hline & Type F & Type D & Type N \\
\hline Large & $100 \mathrm{feet}$ & $70 \mathrm{feet}$ & $70 \mathrm{feet}$ \\
\hline Medium & $70 \mathrm{feet}$ & $50 \mathrm{feet}$ & $50 \mathrm{feet}$ \\
\hline Small & $50 \mathrm{feet}$ & $20 \mathrm{feet}$ & site specific \\
\hline
\end{tabular}

Lake buffer distance depends only on the size of the lake, with different sizes assigned different buffer distances. Lakes greater than eight acres receive a 100 -foot buffer, and lakes between half an acre and eight acres receive a 50-foot buffer. Lakes smaller than half an acre are not assigned a buffer. The streams and lakes data had to be assigned a new field to contain the buffer distance. This field was added to a copy of both of the streams and lake data, so as to not alter the original data. The field was populated using a function that examines the factors involved in the calculation of buffer distance, and then generates the corresponding buffer distance. This new field was then used as the input into the buffer tool. Once the analysis was complete, the copies of the data were deleted.

The physiographic analysis finds areas that have either a slope too steep for harvesting, or have unstable soil types on moderate slopes. Raster datasets of these physiographic features were used in the analysis. Slopes greater than 60 percent are considered too steep for normal harvesting practices. Slopes between 40 and 60 percent are considered too steep when they are composed of erosive or granitic soils. Figure 5-2 illustrate the procedure of identifying these unsuitable areas. 


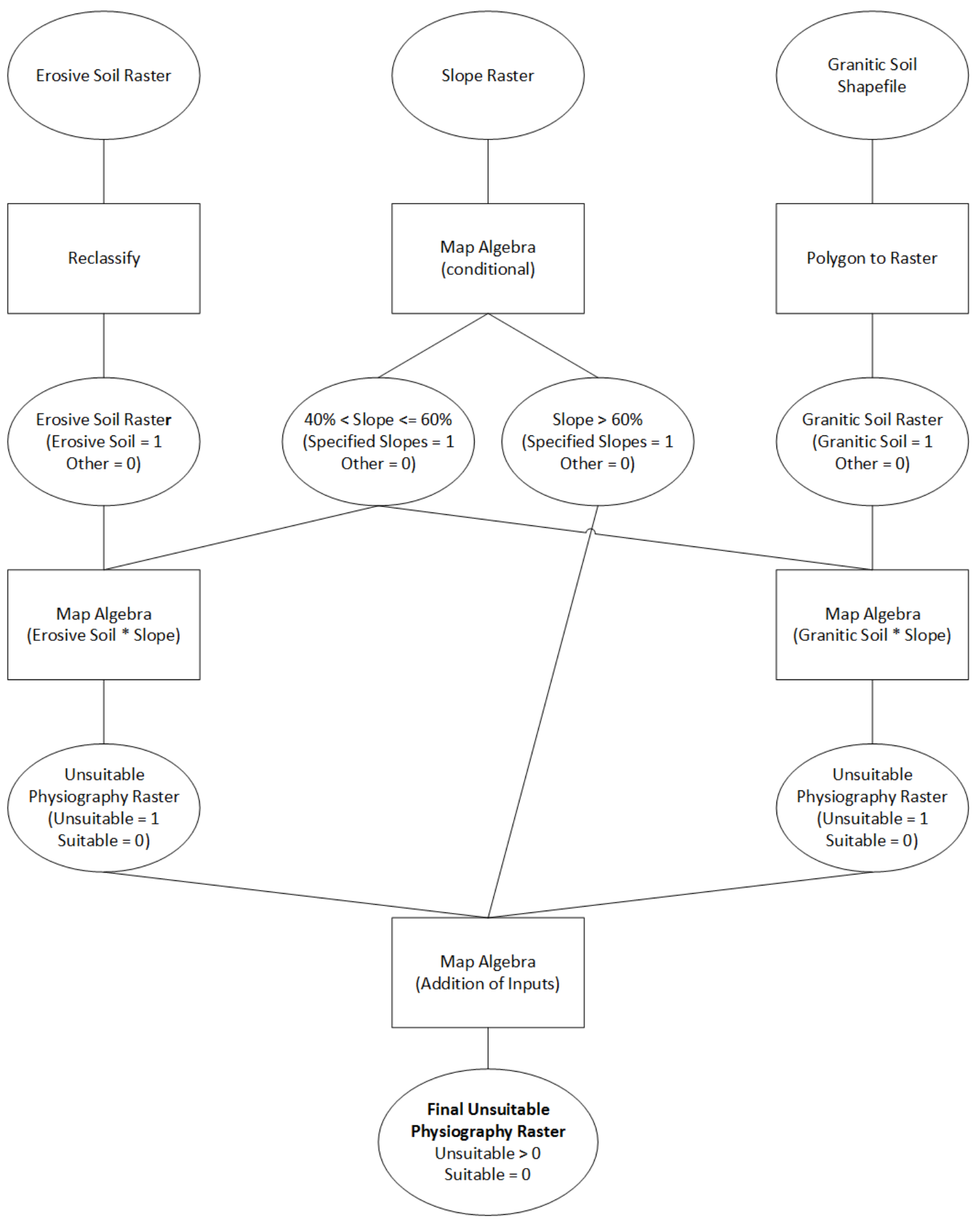

Figure 5-2 Physiographic Analysis Workflow

The soil rasters were classified as having a value of 1 assigned to areas composed of the raster-specific soil types, and a value of 0 applied to all other areas. Two new slope rasters were produced from the original $(40 \%<$ Slope $<=60 \%$, Slope $>60 \%$ ), each classified as having a value of 1 assigned to the specified slope range, and a value of 0 assigned to all other areas. After the classification, the first slope raster was multiplied 
individually with each soil raster using map algebra to identify areas that had slopes between 40 and 60 percent, and were composed of either granitic or erosive soils. This produced two new rasters, which were added together with the steep slopes raster (slope $>60 \%$ ) using map algebra to create a new raster that showed which areas were either too steep, or were of moderate slope and composed of unstable soils. This final raster had values greater than 0 for all physiographically unsuitable areas. Null values were assigned to cells with a value of 0 , and the raster was converted into vector format for use in the final suitable area analysis.

All outputs from the wildlife, public roads, aquatic features, and physiographic analyses depict areas that are not suitable for timber harvesting. These outputs were combined with the merge tool. The output from the merge tool was then input into the erase tool along with the study area boundary. This extracted the unsuitable harvesting locations from the study area, resulting in the ODF compliant timberlands.

\subsection{Generation of Travel Times}

Once the ODF compliant timberlands were identified, travel times to those timberlands were calculated. Again, this workflow was first constructed in ModelBuilder. Once the model was successfully executed, it was turned into a Python script. The script produced from the model had some problems in the network analysis section, and required adjustment to the OD Cost Matrix parameters before the script would run correctly. Figure 5-3 depicts the outline of the travel time generation workflow. 


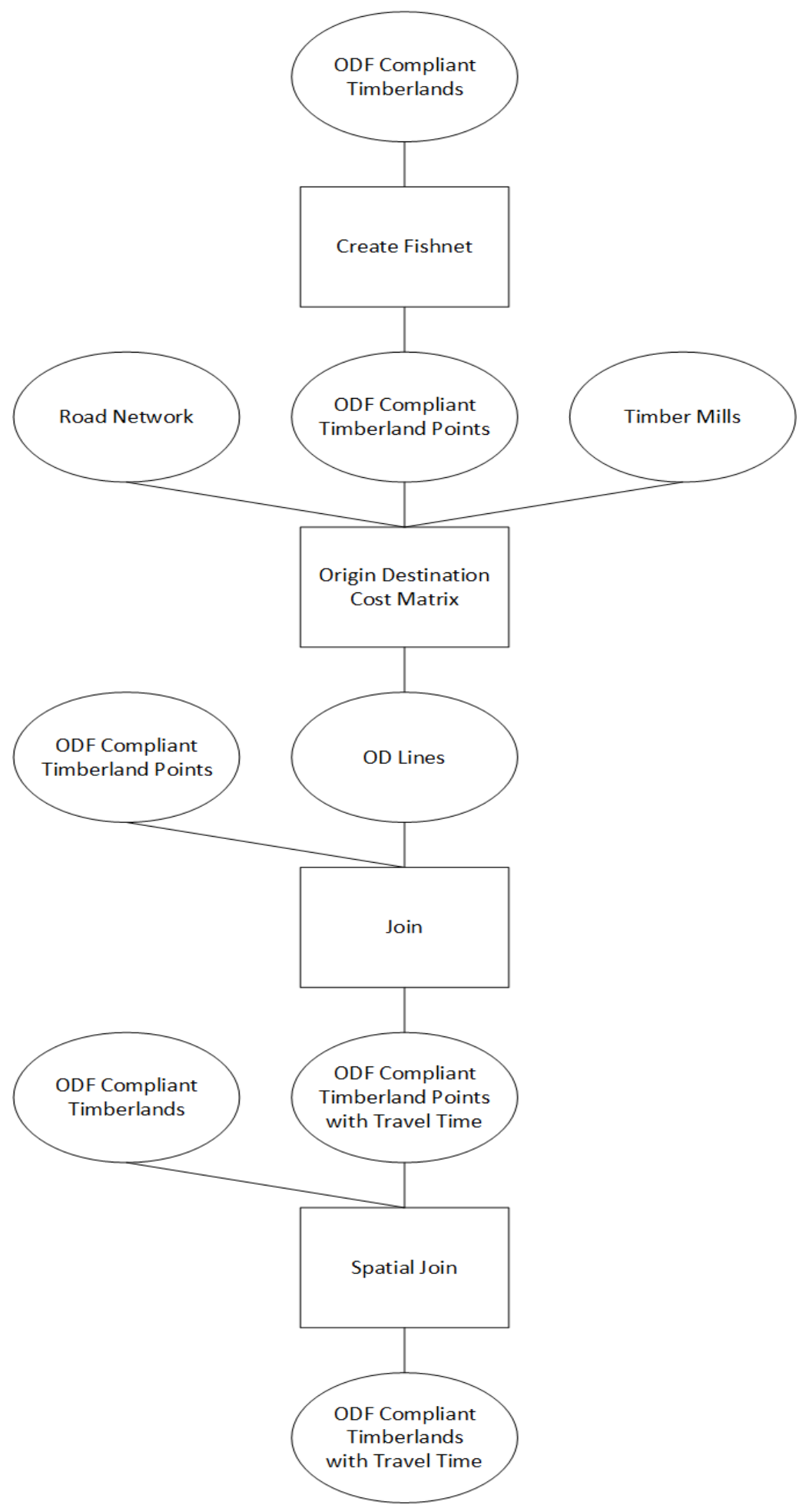

Figure 5-3 Generation of Travel Time

The OD Cost Matrix tool, which generates travel times from origins to destinations, requires origins and destinations as point data. However, the timberlands data had polygonal geometry, and consisted of 46,812 polygons. Many of these polygons were 
quite small, with only six having an area greater than one square mile. While a point could have been generated to represent each polygon and run through the OD Cost Matrix tool, it would be a very computationally demanding process due to the large number of points.

Travel times to polygons that are within one mile of each other will only differ by a small amount, so travel times to polygons within this distance were considered to be the same for the medium scale analysis of this project. To generate points spread out by one mile, the timberlands data were run through the Create Fishnet tool. While one mile was used as the cell width and cell height for this project, the final tool allows the user to specify these parameters. The Create Fishnet tool generates a rectangular pattern of points that match the minimum $\mathrm{X}$, minimum $\mathrm{Y}$, maximum $\mathrm{X}$, and maximum $\mathrm{Y}$ coordinates of the study area, so these points had to be clipped to the timberlands to represent only the study area. This process reduced the number of features required to represent the timberlands from 46,812 polygons to 1,424 point locations separated by one mile. This was much less computationally demanding to process in the OD Cost Matrix tool. Figure 5-4 shows the timberlands with the points generated by this process.

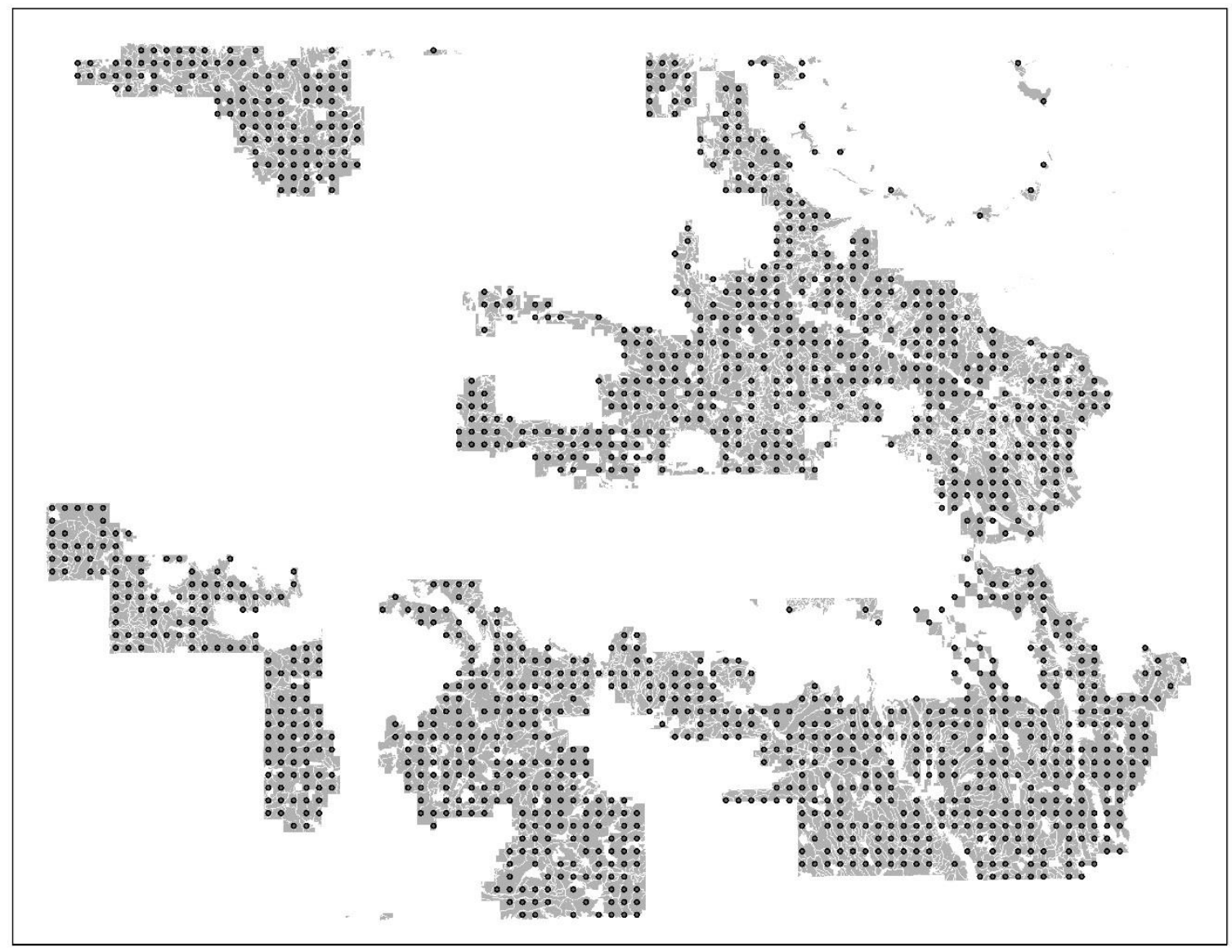

Figure 5-4 Points Generated for Timberlands

These points, along with the timber mill point data and the road network, were then run through the OD Cost Matrix tool. The script prompts the user to enter a field in the timber mill data that specifies the name of the timber mills. The script then uses a for 
loop to run the travel time analysis once for each mill in the timber mill data. For every iteration of the loop, travel times are generated between an individual timber mill and the 1,424 timberlands points. These travel times are produced in a line feature class output from the OD Cost Matrix tool, with each line representing the travel time between the mill and the point in the timberlands. The information from each line is joined to the destination point that it touches. Once each destination point has a travel time joined to it, a spatial join is used to transfer the travel time from the destination points to the polygons of the timberlands. The final step of each for loop iteration is the generation of a feature class by the spatial join tool which contains the spatial information of the timberlands, and has a travel time to a specific timber mill assigned to each polygon. If the input timber mill data contain multiple mills, multiple feature classes will be created, each representing the travel time from all point locations of the timberlands to an individual timber mill.

\subsection{Creation of the Optimal Timberlands with Travel Time Tool}

The scripts for the two main project workflows were combined, and this final script was updated to be compatible with a Python script tool. This process required linking the output of the ODF compliant timberlands workflow to the input of the travel time workflow. The script also needed to be altered to allow users to enter the various datasets and parameters necessary for the analysis. Once these changes were made, a Python script tool was constructed. The user interface for this tool is show in Figure 5-5. 


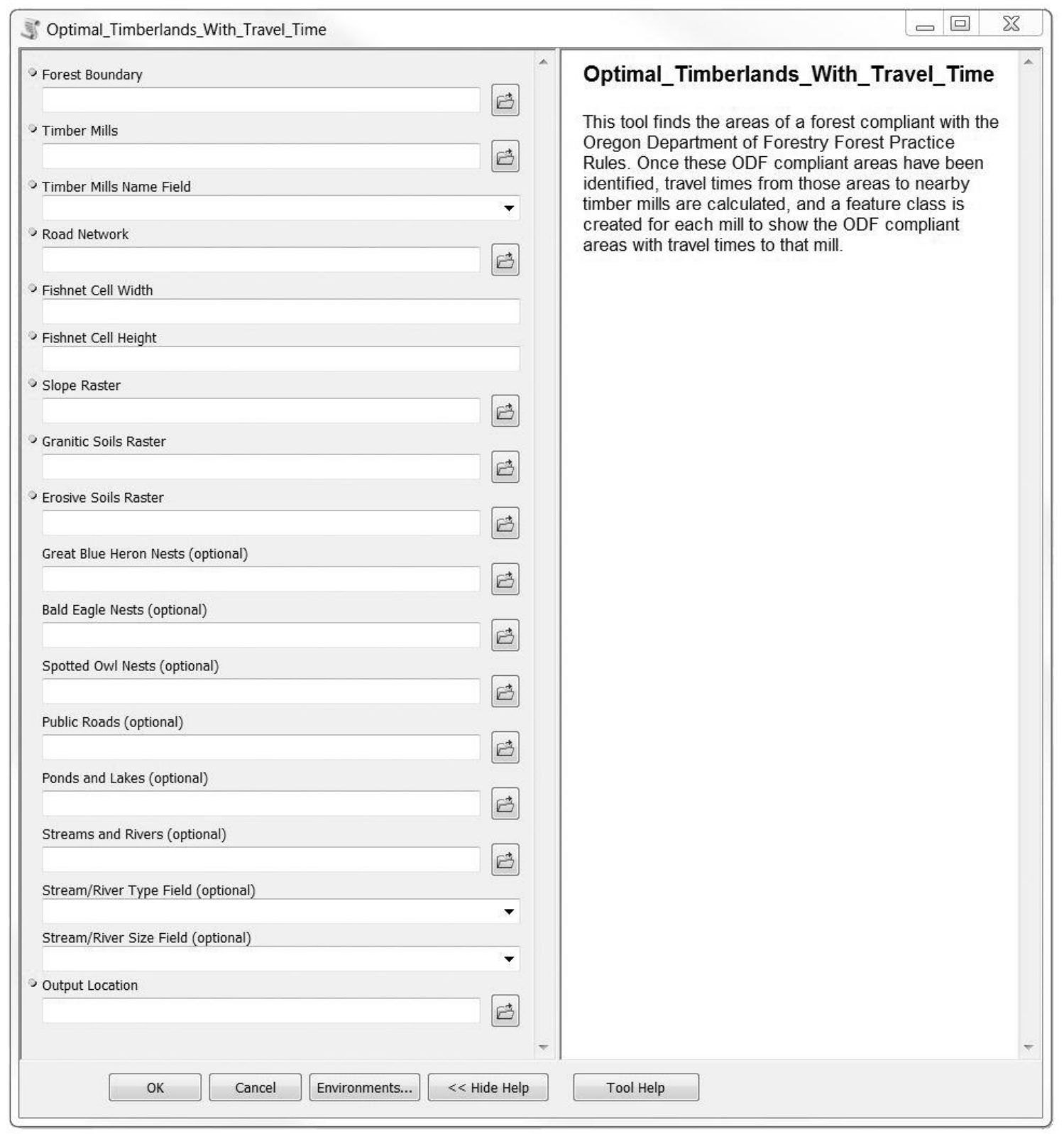

\section{Figure 5-5 Optimal Timberlands with Travel Time Tool Interface}

Some datasets are required for the analysis, and others are optional. These optional datasets are displayed in the tool interface. Each dataset and parameter input field was given a description to help the user clearly understand the required data or parameter entry into the input. The user must specify the output location for the analysis, which must be a geodatabase since the outputs are feature classes. However, the user is not prompted to enter a name for the output, as multiple feature classes may be generated by the analysis. Each output feature class is assigned a name based on the mill name field, which is specified by the user. 


\subsection{Summary}

This chapter provided a description of the process used to create the Optimal

Timberlands with Travel Time tool, including descriptions of the workflow to find the ODF compliant timberlands, the workflow to generate of travel times from these timberlands to nearby timber mills, and the combination of these workflows into a Python script tool. Chapter Six will discuss the results output from this tool. 



\section{Chapter 6 - Results and Analysis}

The first five chapters of this report discussed the needs of the Forest Service to identify travel times to forested areas of the Blue Mountains compliant with Oregon Department of Forestry parameters, as well as the data and methods implemented to generate those results. This chapter discusses the results of the Optimal Timberlands with Travel Time tool built for this project. The first result was the generation of the optimal timberlands from the study area. The second result was a set of travel times generated from the ODF compliant timberlands to nearby timber mills.

\subsection{ODF Compliant Timberlands}

The project study area covers 1,617 square miles of mountainous forested terrain. The study area data were run through the Optimal Timberlands with Travel Time tool and only 1,391 square miles were found to be compliant with the ODF Forest Practice Rulebook criteria. These criteria included locations of wildlife, streams, lakes, public roads, and physiography. Figure 6-1 shows the ODF compliant timberlands and nonharvestable areas.

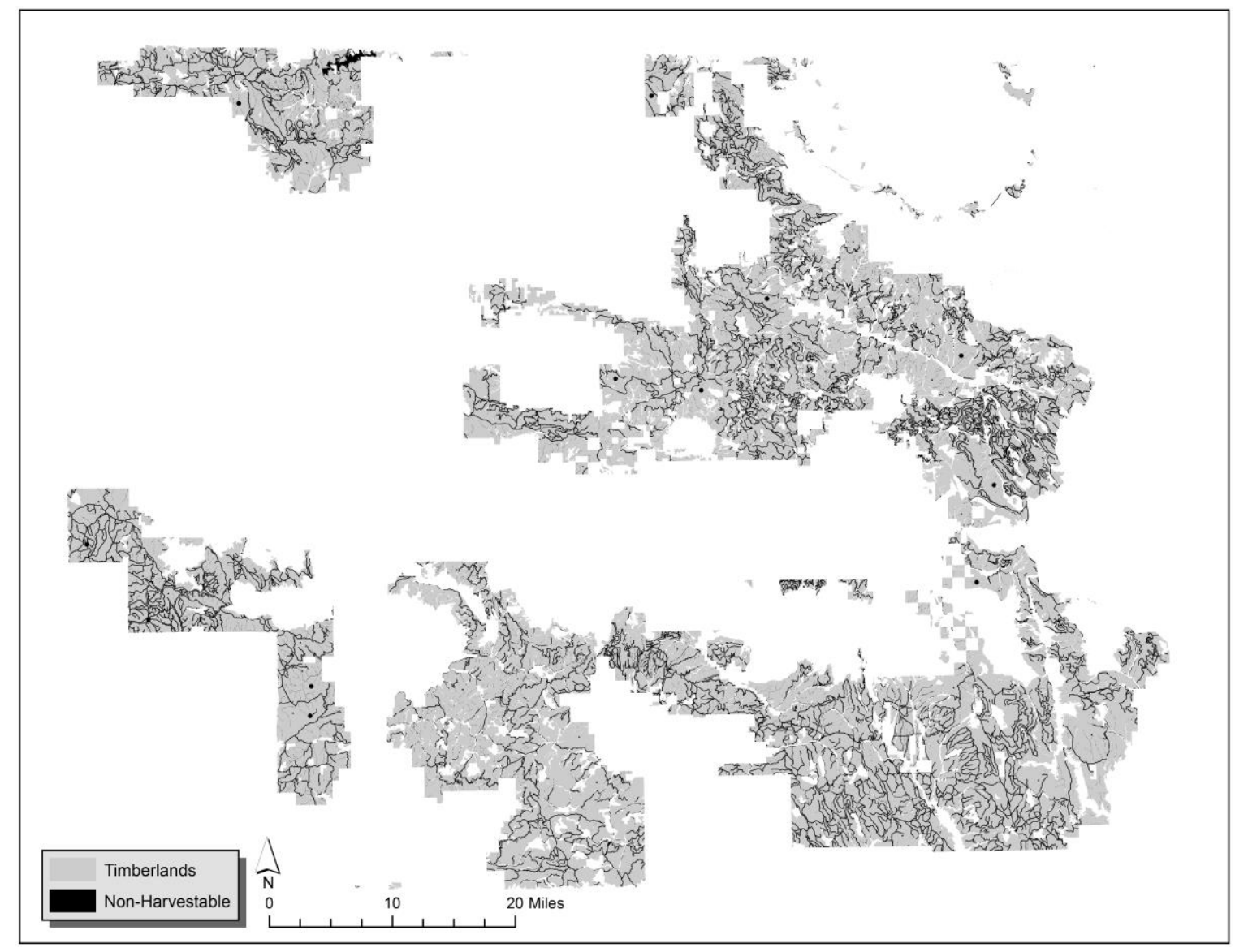

Figure 6-1 ODF Compliant Timberlands and Non-Harvestable Areas 
Roads generated the greatest non-harvestable areas, with 249 square miles unable to be harvested due to their close proximity to roads. Seventeen square miles were found to be non-harvestable due to stream proximity. About two square miles were unsuitable due to physiography, where slopes were too steep, or moderate but with unstable soils. Wildlife buffers generated 1.58 non-harvestable square miles. However, this number was generated from artificial wildlife data, so the true number would be different. Lakes accounted for the smallest non-harvestable area, covering only .125 square miles.

When all of these non-harvestable areas were added, they comprised 269 square miles. However, 43 square miles of this area contained overlapping features. This was primarily due to roads and/or streams located closer together than their respective buffer distances, so buffers of these features overlapped. When two buffers overlapped, they were treated as the same area by the Erase tool. Overlapping buffered areas were erased only once from the study area, resulting in a total non-compliant area of 226 square miles rather than 269 square miles.

\subsection{Travel Times from Timberlands to Mills}

After generating the ODF compliant timberlands, the Optimal Timberlands with Travel Time tool generated travel times from the timberlands to nearby timber mills. Travel time analysis was conducted using the OD Cost Matrix tool, which generates travel times under the assumption that a vehicle can travel between two locations at the exact speed limit for each road segment of the route, from start to finish. Thirty-two mills which were within 75 miles of the timberlands were used in this analysis (Figure 6-2). These mills were geographically spread out and the study area was large, so travel times varied widely. 


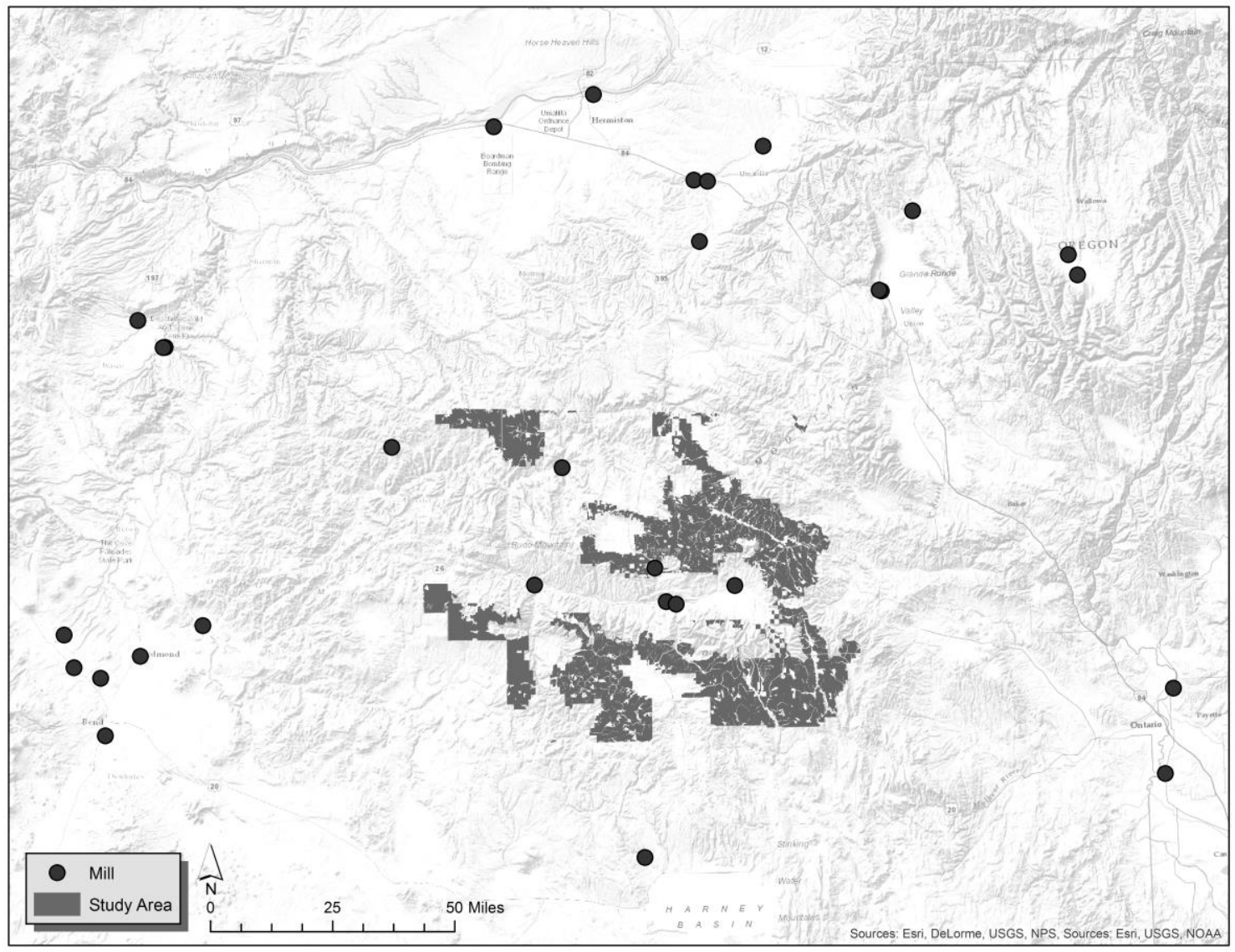

Figure 6-2 Timber Mills Near Study Area

The mean travel time between all mills and the study area was 214 minutes, which was calculated as the mean of all timber mills' mean travel time to all forest polygons. The closest timber mill to the study area, Juniper Plus Inc., had a lowest travel time of less than a minute, which represents an artificial network travel time. This number is unrealistically low, as a real world vehicular travel time would be several minutes longer due to acceleration, deceleration, traffic, and stops. The highest travel time was 443 minutes, which was to JayZee Lumber. Specific results for three particular timber mills are discussed in this section.

\subsubsection{Juniper Plus Inc.}

Located only 226 feet from the border of the study area, the Juniper Plus mill is in a prime location to serve central and north-eastern areas of the timberlands. The shortest travel time for this mill was less than a minute. The greatest travel time was 183 minutes, which is to access the south eastern corner of the timberlands. The mean travel time to all polygons of the timberlands was 89 minutes. Results for this mill are displayed in Figure 6-3. 


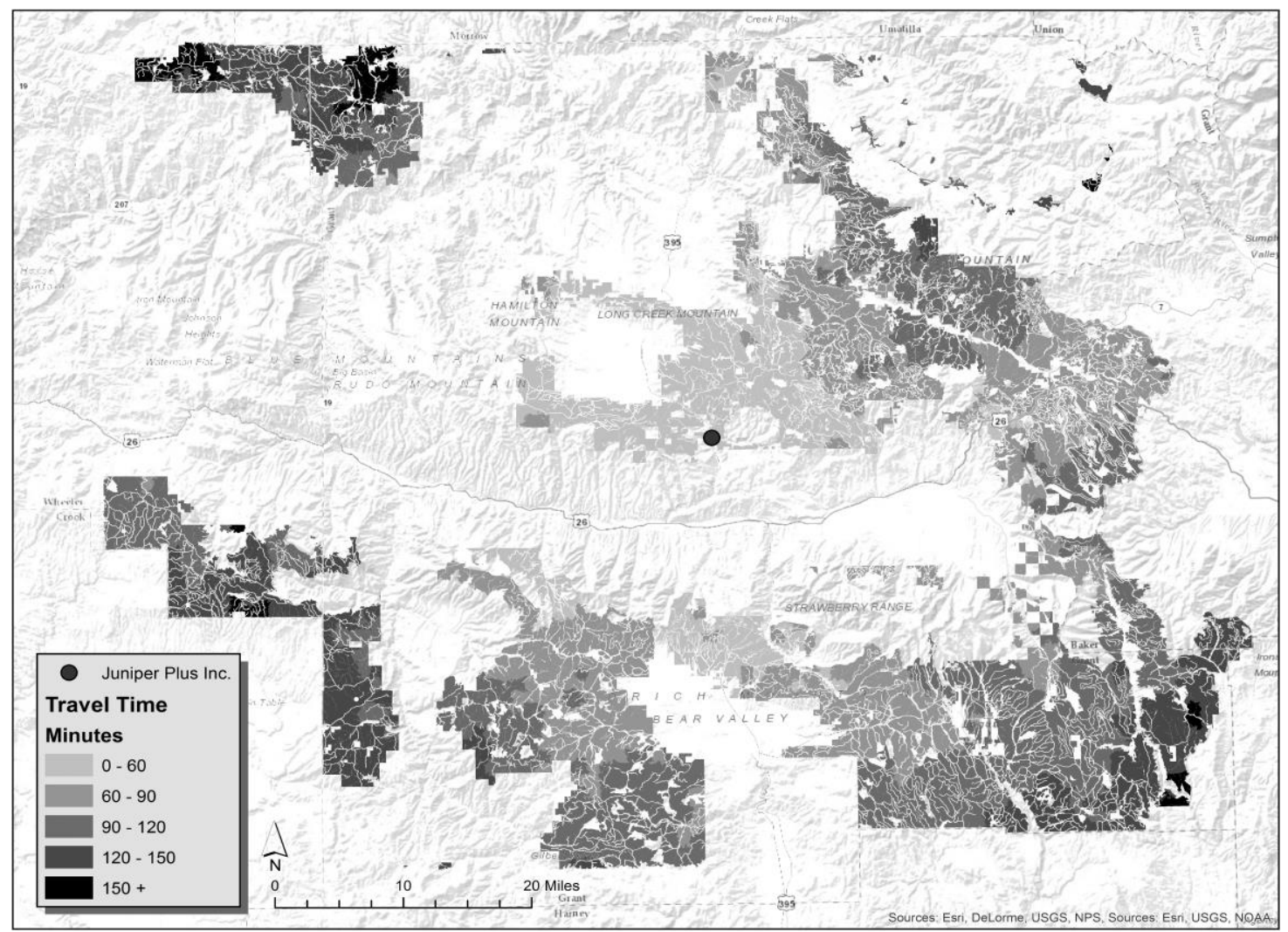

Figure 6-3 Travel Times for Juniper Plus Inc.

While this mill was well placed to serve the central and northeastern parts of the study area, it had higher travel times to the southern and western areas. These areas were better served by other mills.

\subsubsection{Joseph's Juniper Inc.}

Located 23 miles south of the study area, Joseph's Juniper Inc. was well placed to serve the southern parts of the study area. While it was only 23 miles away, the shortest network distance to reach the study area by road was roughly 50 miles. Many of these were mountain roads with lower speed limits, so the lowest travel time for this mill was 85 minutes. The highest travel time was 291 minutes, which was to access the northwestern part of the study area. The mean travel time was 168 minutes. Results for this mill are displayed in Figure 6-4. 


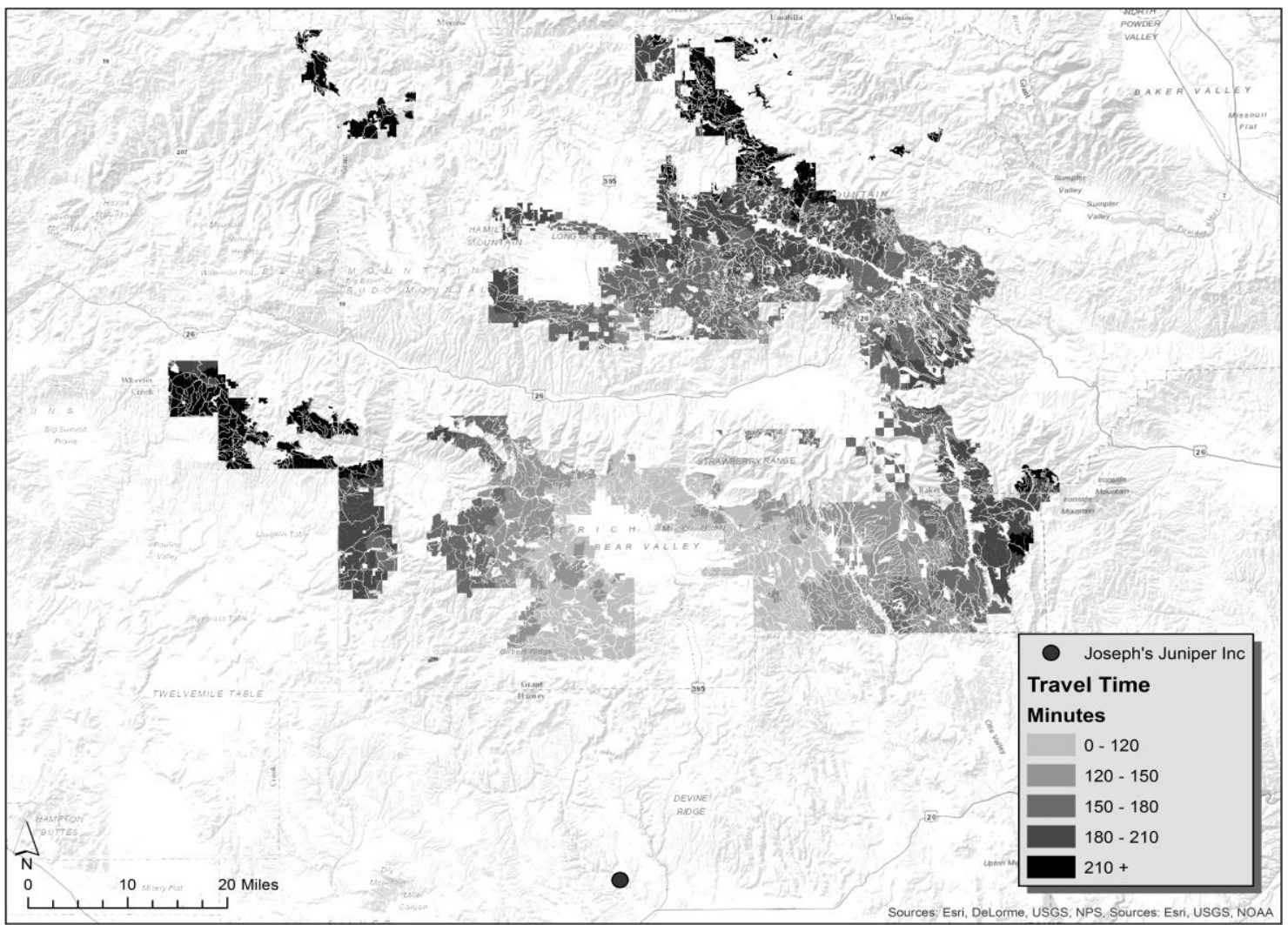

Figure 6-4 Travel Times for Joseph's Juniper Inc.

As with Juniper Plus Inc., travel times from this mill to the northwestern section of the study area were high. The best mill to access these northwestern areas was Juniper Log Homes.

\subsubsection{Juniper Log Homes}

Located 10 miles to the west, Juniper Log Homes is well suited to serve the northwestern parts of the study area. The shortest network distance to reach the study area was 19 miles, and travel time to this location was 49 minutes. The shortest overall travel time was 47 minutes. The longest travel time was 307 minutes, which was to access a polygon in the south eastern corner of the study area. Results for this mill are presented in Figure 6-5. 


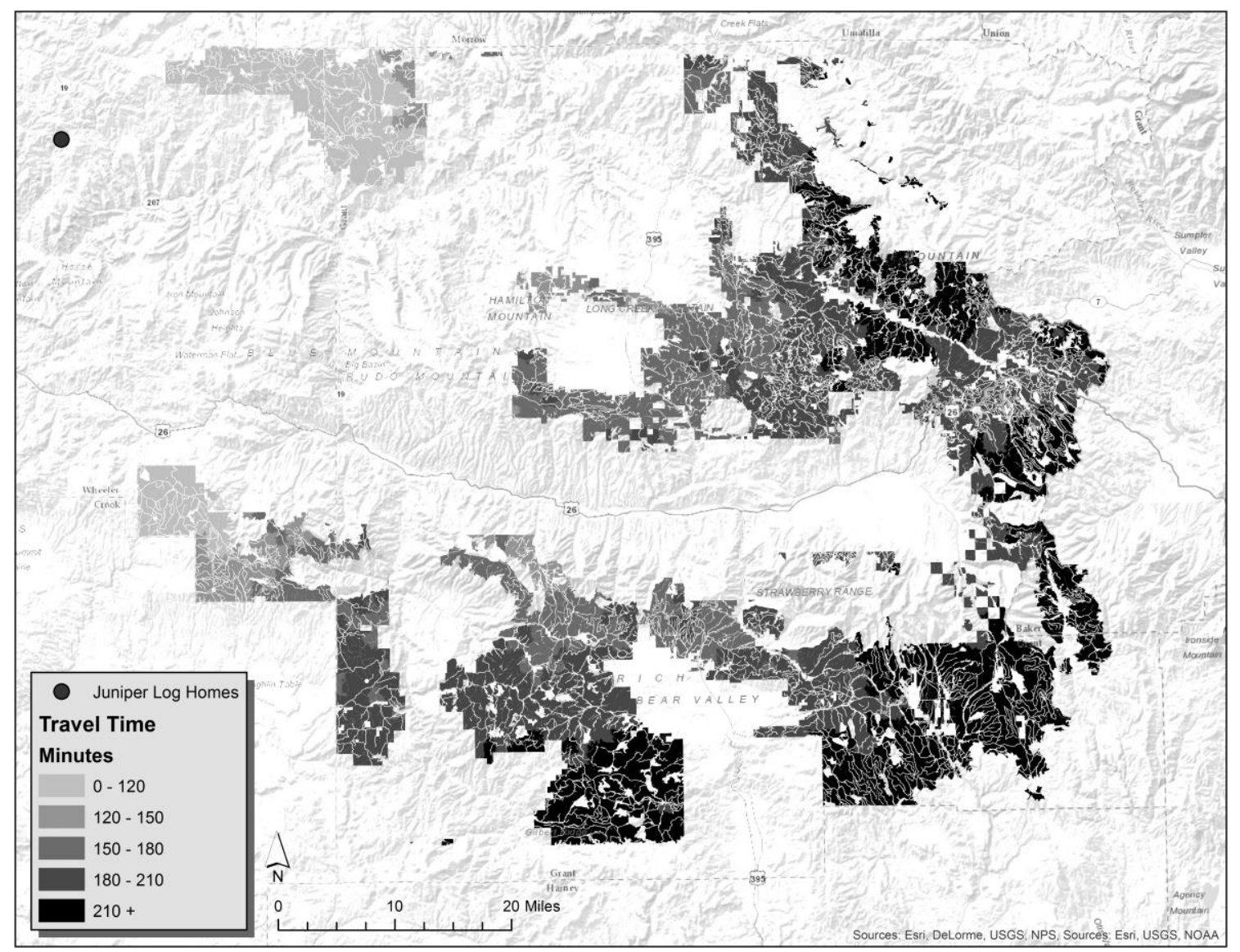

Figure 6-5 Travel Times for Juniper Log Homes

While Juniper Log Homes was located close to the northwestern part of the study area, it was relatively far from the rest of the study area. At 190 minutes, the mean travel time for this mill was the highest of the three mills examined in this section. While it would likely not service a large portion of the study area, it is one of the best suited mills to service the northwestern island of the study area.

\subsection{Travel Time Analysis Methodology Testing}

The purpose of generating travel times to a set of fishnet cells instead of to each individual study area polygon was to save execution time. This was based off the assumption that time would be saved without sacrificing accuracy of results. To test this assumption, travel times were generated from the Juniper Plus mill to 10 random points throughout the study area. This test was conducted for five different fishnet cell sizes: 0.5 miles, 1 mile, 2 miles, 5 miles, and 10 miles. These results were then compared to the true travel times for the 10 points, and the percent error was calculated. Results from this test can be seen in Table 6-1. 
Table 6-1 Travel Time Test Results

\begin{tabular}{|c|c|c|c|c|c|c|c|c|c|c|c|c|}
\hline & & & & & Fis! & hnet size & & & & & & \\
\hline & & .5 Miles & & 1 Mile & & $\underline{2 \text { Miles }}$ & & $\underline{5 \text { Miles }}$ & & $\underline{10 \text { Miles }}$ & & True Time \\
\hline & & Minutes & $\%$ Error & Minutes & $\%$ Error & Minutes & $\%$ Error & Minutes & $\%$ Error & Minutes & $\%$ Error & Minutes \\
\hline & 1 & 134 & 0.8 & 133 & 0.0 & 133 & 0.0 & 119 & 10.5 & 77 & 42.1 & 133 \\
\hline & 2 & 47 & 14.5 & 47 & 14.5 & 53 & 3.6 & 52 & 5.5 & 62 & 12.7 & 55 \\
\hline & 3 & 110 & 0.9 & 110 & 0.9 & 105 & 5.4 & 103 & 7.2 & 92 & 17.1 & 111 \\
\hline & 4 & 124 & 0.0 & 125 & 0.8 & 121 & 2.4 & 119 & 4.0 & 77 & 37.9 & 124 \\
\hline Polygon & 5 & 75 & 0.0 & 75 & 0.0 & 71 & 5.3 & 82 & 9.3 & 77 & 2.7 & 75 \\
\hline & 6 & 115 & 5.5 & 108 & 0.9 & 105 & 3.7 & 109 & 0.0 & 125 & 14.7 & 109 \\
\hline & 7 & 112 & 4.7 & 107 & 0.0 & 108 & 0.9 & 102 & 4.7 & 103 & 3.7 & 107 \\
\hline & 8 & 21 & 16.0 & 28 & 12.0 & 25 & 0.0 & 22 & 12.0 & 62 & 148.0 & 25 \\
\hline & 9 & 130 & 0.0 & 135 & 3.8 & 138 & 6.2 & 135 & 3.8 & 119 & 8.5 & 130 \\
\hline & 10 & 88 & 0.0 & 83 & 5.7 & 90 & 2.3 & 107 & 21.6 & 82 & 6.8 & 88 \\
\hline & an: & & 4.24 & & 3.87 & & 2.98 & & 7.87 & & 29.42 & \\
\hline
\end{tabular}

Small fishnet sizes were expected to produce more accurate results, due to the increased density of points. However, accuracy increased slightly as the fishnet cell size grew from .5 miles to 2 miles. Starting at a 2 miles, the accuracy decreased as the cell size grew to 10 miles. The .5 mile, 1 mile, and 2 mile fishnets all had a mean travel time percent error less than five percent. Errors varied by less than 1.5 percent among these three, so the accuracy of their results were similar. With a fishnet size extended out to 5 miles, the percent error grows to 7.87. The 10-mile fishnet had a percent error of 29.42, which is relatively large. Drawing a cut off point for acceptable percentage error depends on the use of the results. When accuracy is more vital, smaller percentage errors are required, and fishnet size should be 2 miles or less.

Along with accuracy, the speed of execution of the analysis for the different fishnet sizes was also examined. Execution time for one timber mill at each of the different fishnet sizes is listed in Table 6-2.

Table 6-2 Execution Time per Individual Timber Mill

\begin{tabular}{|c|c|c|c|c|c|c|}
\hline \multicolumn{7}{|c|}{ Fishnet Size } \\
\hline \multirow{4}{*}{ Run Time } & .5 Miles & 1 Mile & 2 Miles & 5 Miles & 10 Miles & No Fishnet \\
\hline & $1: 52$ & $1: 35$ & $1: 40$ & $1: 50$ & $2: 07$ & $5: 11$ \\
\hline & $1: 51$ & $1: 35$ & $1: 39$ & $1: 50$ & $2: 08$ & $5: 10$ \\
\hline & $1: 52$ & $1: 36$ & $1: 41$ & $1: 49$ & $2: 08$ & $5: 10$ \\
\hline Average: & $1: 52$ & $1: 35$ & $1: 40$ & $1: 50$ & $2: 08$ & $5: 10$ \\
\hline
\end{tabular}

Execution times were expected to decrease as the fishnet cell size increase, since larger fishnet cell size results in fewer fishnet points to process. However, test results were not conclusive, and indicated the opposite was true. When increasing cell size from .5 miles to 1 mile, the execution time per mill dropped by 17 seconds on average. However, when increasing fishnet cell size beyond 1 mile, execution time increased, maxing out a 2:08 per mill for a 10-mile fishnet. While results among the tested fishnet 
cell sizes varied, they were spread out by less than 35 seconds on average. When all 46,000 points of the study area were run through the tool, the execution time per mill was 5:10, which is more than 3 minutes higher than the highest tested fishnet execution time. This indicates that the process of using a fishnet to calculate travel times does indeed reduce execution time of the Optimal Timberlands tool.

The project analysis was conducted at a fishnet cell size of 1 mile. According to the tests, the travel time results of analysis at this cell size have an average percentage error 3.87. The execution time per mill was 1:35, which saves 3:35 per mill. With 32 mill used for the analysis, this saved a total of 74 minutes of execution time with only a small reduction in accuracy.

\subsection{Summary}

This chapter examined the results output from the Optimal Timberlands with Travel Time tool. Of the 1,617 square miles of the original study area, 1,391 square miles were found to be compliant with ODF standards. Travel times to these compliant timberlands were calculated for 32 timber mills located within 75 miles of the timberlands. The lowest overall travel time was less than a minute, and the highest was 443 minutes, and the overall mean travel time was 214 minutes. Three timber mills that were best suited to service the study area were Juniper Plus Inc., Joseph's Juniper Inc., and Juniper Log Homes. Tests were conducted to judge the accuracy and efficiency of different fishnet cell sizes input into the tool, finding that a one-mile fishnet saved processing time without greatly altering the accuracy of results for the scale of this project. However, recommended fishnet size for future use of this tool depends on the scale of the analysis: one mile for local analysis; five miles for regional analysis; ten miles for national analysis. Further conclusions and future work are discussed in the next chapter. 


\section{Chapter 7 - Conclusions and Future Work}

The previous chapter discussed the results output from the Optimal Timberlands with Travel Time tool. This chapter discusses conclusions and future work that could be built upon this project. The project was able to satisfy the requirements of the client, and the tool output from the project is ready to be deployed by the client on future projects.

\subsection{Conclusion}

The overall goal of this project was to find areas within a specific forest boundary that could be harvested for timber in order to raise profits for forest restoration activities. Meeting this goal involved collecting data from the client and a variety of government sources, using the data to identify forested areas compliant with ODF standards, and identifying vehicular travel times from the ODF compliant timberlands to nearby timber mills. A Python script was developed which integrated the necessary geoprocessing tools and parameters used to identify the ODF compliant timberlands and in the calculation of travel times. This script was then turned into a custom Python script tool which allows the user to enter the datasets and parameters necessary for the analysis. This tool-the Optimal Timberlands with Travel Time tool—outputs one feature class showing travel times to the different areas of the input timberlands for each mill input into the tool. This tool meets the requirements provided by the client at the beginning of the project.

\subsection{Future Work}

The Optimal Timberlands with Travel Time tool identifies areas of a forest that are compliant with the Oregon Department of Forestry's Forest Practice Rulebook. This tool could be customized to find areas compliant with the forest practice rules of other states. A significant challenge would be to develop a tool that could identify compliant forested areas for multiple states, using parameters from their individual rulebooks. A user could choose which state's rules would be implemented by the tool, and different results would be output based on which state was chosen by the user.

The travel times identified by this tool were based on network distance and speed limit. The travel times assigned to the polygons of the study area were based on the routes that have the lowest overall travel time. However, other factors can affect which route would be optimally chosen to access a particular polygon. These factors include road owner, road width, road surface, and slope. Raster-based cost distance tools could be used in place of the network analyst tool to incorporate these additional factors in the selection of an optimal route, and travel times from timberlands to timber mills could then be based on those routes.

Individual timber mills will have varying types of equipment for processing timber. Diverse sizes and types of saw blades are used to process different sizes and species of trees. If timber mill data contained information about which types of timber are processed at the mills, and the polygons of the timberlands included information about the sizes and 
species of trees on each site, the project tool could be customized to find which timber mills are capable of processing the timber extracted from the different polygons of the timberlands. Travel times for individual timber mills could be calculated for only those timberland areas that contain timber compatible with each individual timber mill.

\subsection{Summary}

Using Python scripting, a tool was developed to help the USDA Forest Service locate areas of the Blue Mountains of Oregon that could be harvested for timber to produce profits used to aid in the restoration of those forests. This involved finding forested areas compatible with the Oregon Department of Forestry's Forest Practice Rules, and then generating travel times from the compliant areas to nearby timber mills. Future work could identify timberlands compliant with the forest practice rules of other states. The tool could also be amended to include additional factors in the calculation of travel times to the compliant timberlands. Another possible update to the tool would be the consideration of which sites have timber compatible with individual mills when calculating travel times. The tool designed for this project successfully fulfilled the requirements outlined by the client. 


\section{Work Cited}

Anderson, G., Flisberg, P., Liden, B., \& Ronnqvist, M. (2008). RuttOpt - a decision support system for routing of logging trucks. Canadian Journal of Forest Research, 38, 1784-1796. doi:10.1139/X08-017

Antikainen, H. (2013). Comparison of Different Strategies for Determining Raster-Based Least-Cost Paths with a Minimum Ammount of Distortion. Transactions in GIS, 17(1), 96-108.

Caissie, D. (2006). The thermal regime of rivers: a review. 51, 1389-1406. doi:10.1111/j.1365-2427.2006.01597.x

Choi, Y., Um, J.-G., \& Park, M.-H. (2013, 10 30). Finding Least-Cost Paths Across a Continuous Raster Surface with Discrete Vector Networks. Cartography and Geographic Information Science, 41(1), 75-85.

Dean, D. (1997). Finding Optimal Routes for Networks of Harvest Site Access Roads using GIS-based Techniques. Canadian Journal of Forest Research, 27, 11-22.

Delamater, P., Messina, J., Shortridge, A., \& Grady, S. (2012). Measuring geographic access to health care: raster and network-based methods. International Journal of Health Geographics, 11(15), 1-18. doi: 10.1186/1476-072X-11-15

Delvin, G. J., McDonnell, K., \& Ward, S. (2008, January). A Study of Travel Times and Distances for Haulage Routes in Ireland using GPS and GIS. Journal of Transport Geography, 16(1), 63-72.

Dietrich, W., \& Dunne, T. (1978). Sediment Budget for a Small Catchement in Mountainous Terrain. 29, 191-206.

Egan, T. (1990, June 23). U.S. Declares Owl To Be Threatened By Heavy Logging. The New York Times.

Ghasemi, S. (2015, July 6). A rapid assessment of logging effects on bird species in Kelantan Forests Areas, Malaysia. PeerJ PrePrints.

Gonclaves, A. B. (2010). An Extension of GIS-Based Least-Cost Path Modelling to the Location of Wide Paths. International Journal of Geographical Information Science, 24(7), 983-996.

Grieg, S., Sear, D., \& Carling, P. (2005). The impact of fine sediment accumulation on the survival of incubating salmon progeny: Implications for sediment management. 344, 241-258. doi:10.1016/j.scitotenv.2005.02.010

Imaizumi, F., Sidle, R., \& Kamei, R. (2008). Effects of forest harvesting on the occurrence of landslides and debris flows in steep terrain of central Japan. 33, 827-840.

Jordan, P. (2006). The use of sediment budget concepts to assess the impact on watersheds of forestry operations in the southern interior of British Columbia. 79, 27-44.

Kreutzweiser, D. C., \& Holmes, S. (2009). Stream temperature response to partial-harvest logging in riparian buffers of boreal mixedwood forest watersheds. 39, 497-506. doi:10.1139/X08-191

Ling, T.-Y., Soo, C.-L., Sivalingam, J.-R., Nyanti, L., Sim, S.-F., \& Grinang, J. (2016). Assessment of the Water and Sediment Quality of Tropical Forest Streams in 
Upper Reaches of the Baleh River, Sarawak, Malaysia, Subjected to Logging Activities. 2016, 1-13.

Loreno, S. (2014). The Cost of Hauling Timber: A Comparison of Raster-and VectorBased Travel Time Estimates in GIS. Portland: Portland State University.

ODF. (2014, January). Forest Practice Administrative Rules and Forest Practices Act. Forest Practice Administrative Rules and Forest Practices Act, 94. Oregon, United States: Oregon Department of Forestry.

Ramezani, J., Rennebeck, L., Closs, G., \& Matthael, C. (2014). Effects of fine sediment addition and removal on stream invertebrates and fish: a reach-scale experiment. 59, 2584-2604. doi:10.1111/fwb.12456

Tobias, J. (2015, July 9). Hidden impacts of logging. Nature, 523, 163-164. 


\section{Appendix A. Python Script}

\# Import arcpy module

import arcpy, sys, os

from arcpy.sa import *

arcpy.CheckOutExtension("Spatial")

import arcpy.na

arcpy.CheckOutExtension("Network")

\#Allow the script to overwrite existing outputs

arcpy.env.overwriteOutput $=$ True

\#Identify Unsuitable Harvest Areas

arcpy.AddMessage("Identifying Unsuitable Timber Harvest Areas")

print ("Identifying Unsuitable Harvest Areas")

\#Input Foresty Boundaries

forestBoundary $=$ arcpy.GetParameterAsText $(0)$

\#Create List of Unsuitable Areas

unsuitableAreas = []

\#Wildlife Buffers

print ("Wildlife Buffers")

arcpy.AddMessage("Wildlife")

\#Great Blue Heron

Great_Blue_Heron $=$ arcpy.GetParameterAsText $(1)$

if Great_Blue_Heron:

Great_Blue_Heron_buffer = "in_memoryl|Great_Blue_Heron_buffer"

arcpy.Buffer_analysis(Great_Blue_Heron, Great_Blue_Heron_buffer, "300 Feet",

"FULL", "ROUND", "NONE", "", "GEODESIC")

unsuitableAreas.append(Great_Blue_Heron_buffer)

\#Bald Eagle

Bald_Eagle $=$ arcpy.GetParameterAsText $(2)$

if Bald_Eagle:

Bald_Eagle_buffer = "in_memory $\mid$ Bald_Eagle_buffer"

arcpy.Buffer_analysis(Bald_Eagle, Bald_Eagle_buffer, "330 Feet", "FULL",

"ROUND", "NONE", "", "GEODESIC")

unsuitableAreas.append(Bald_Eagle_buffer)

\#Spotted Owl

Spotted_Owl = arcpy.GetParameterAsText(3) 


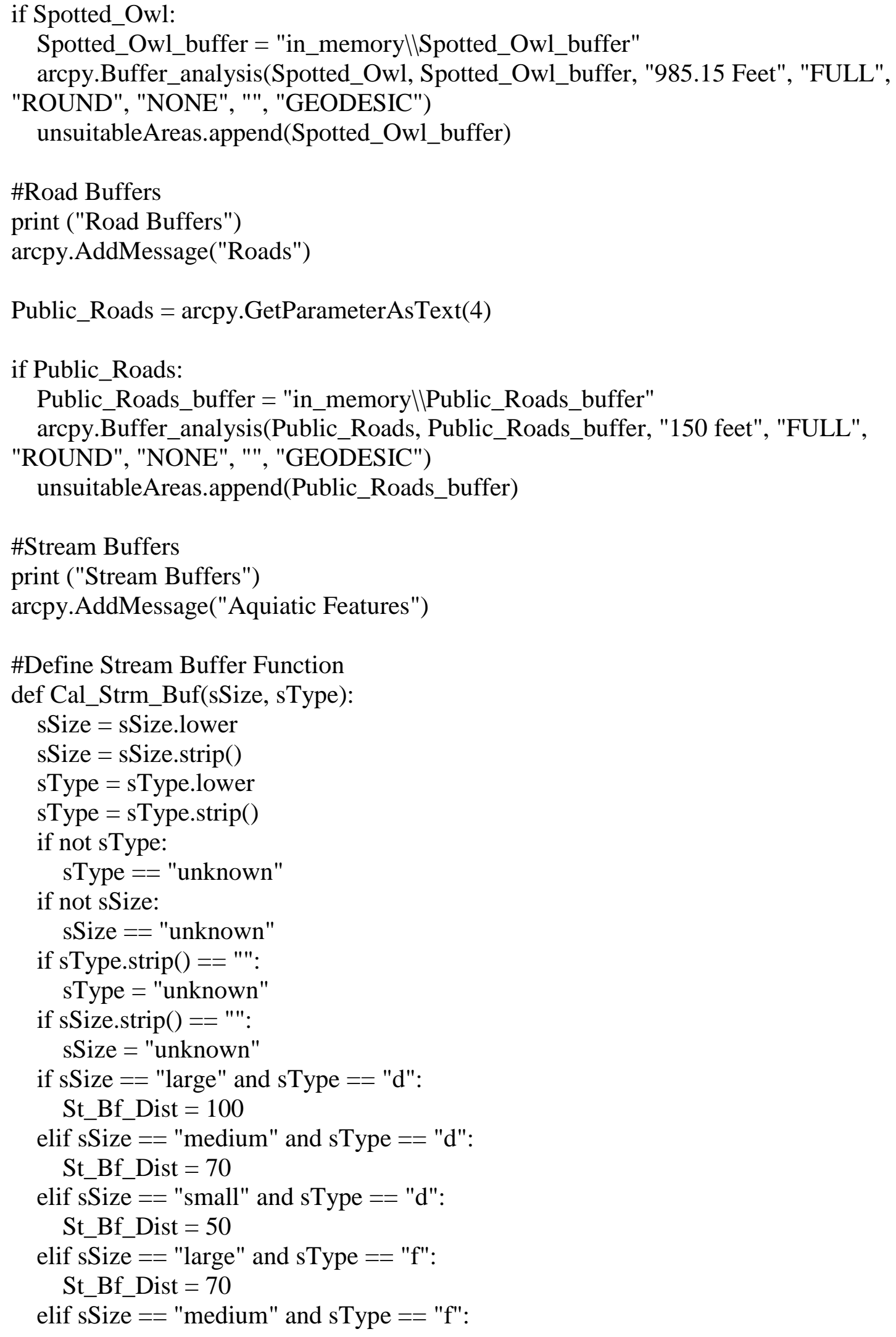




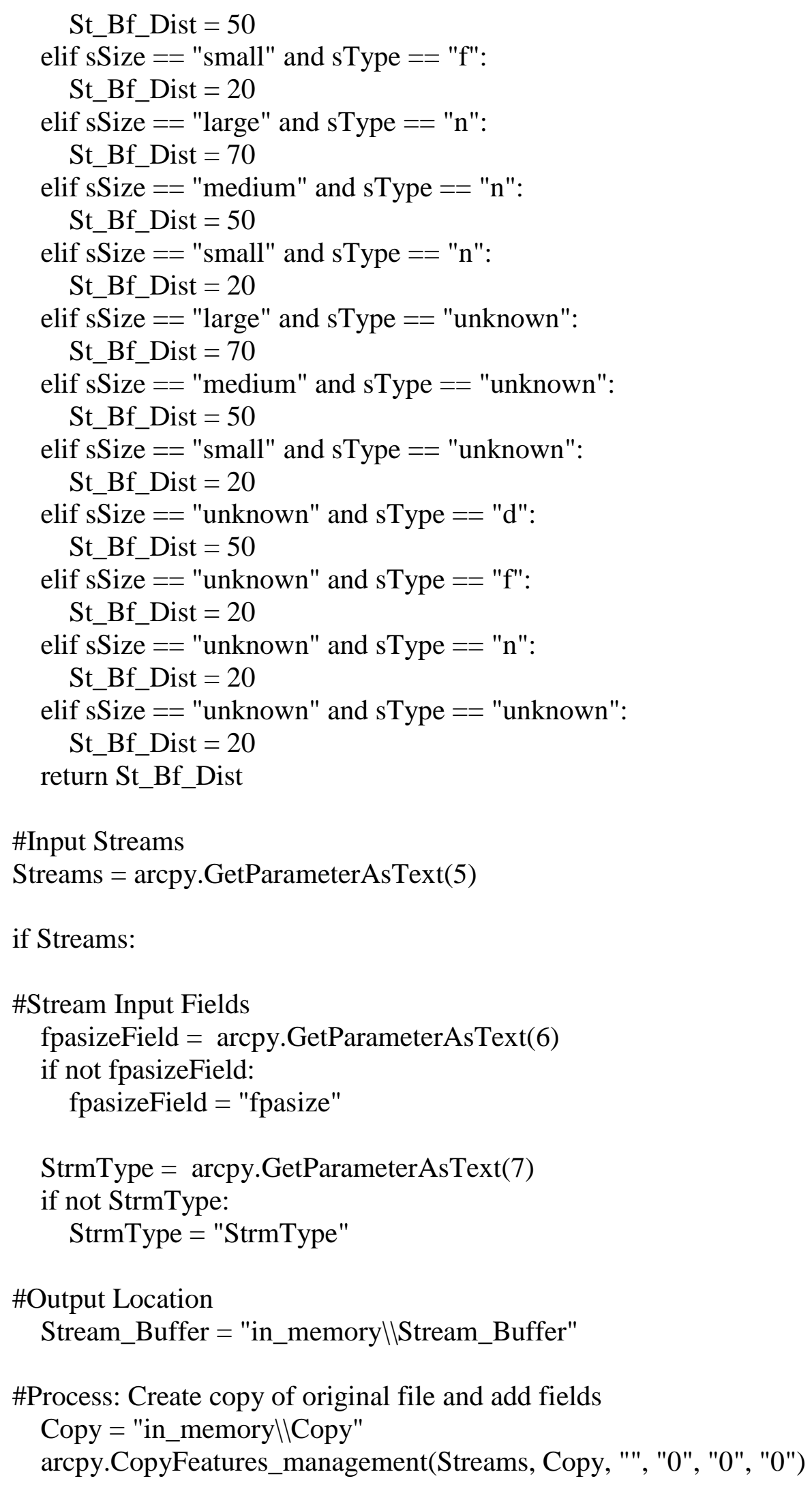




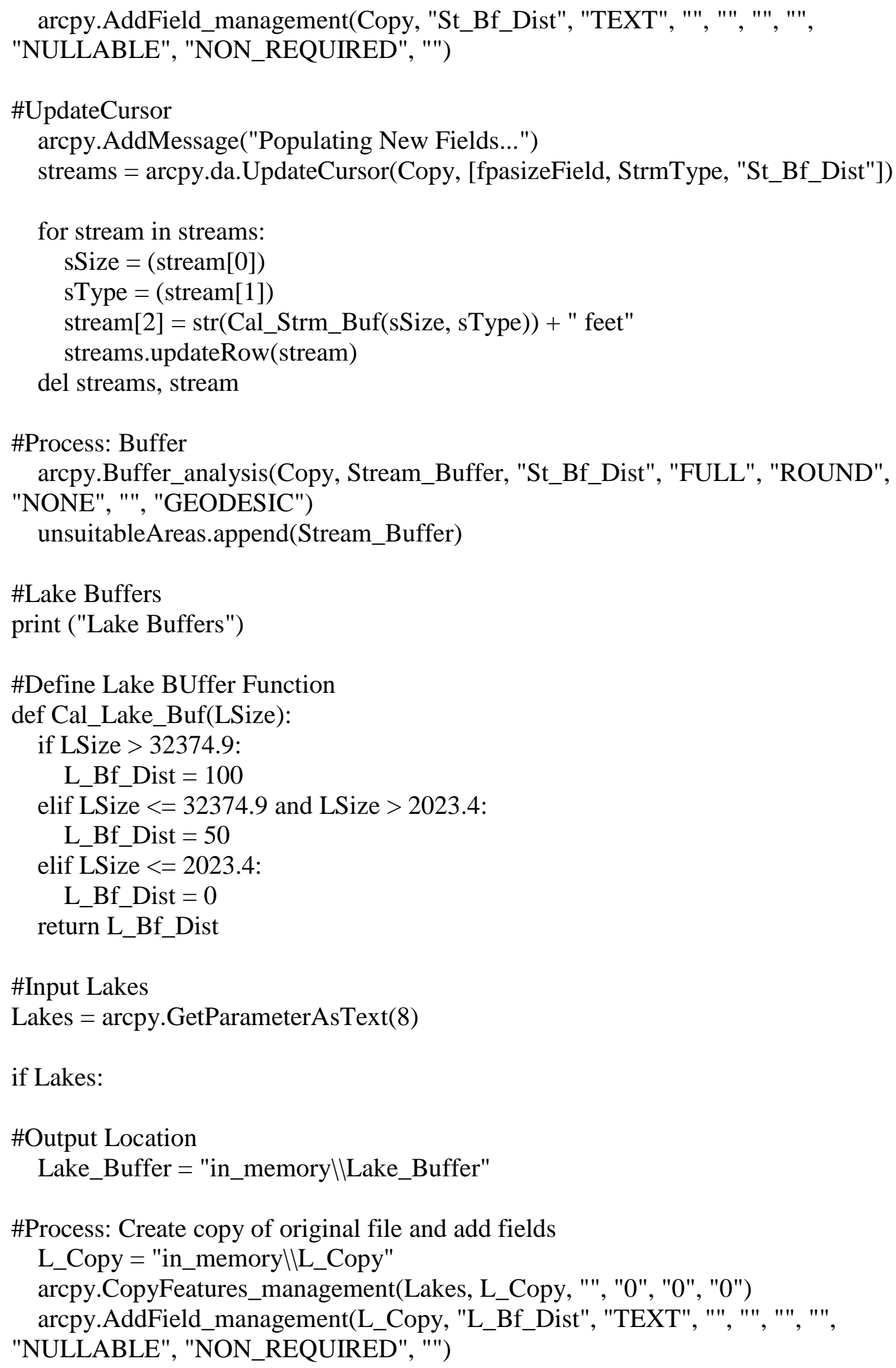




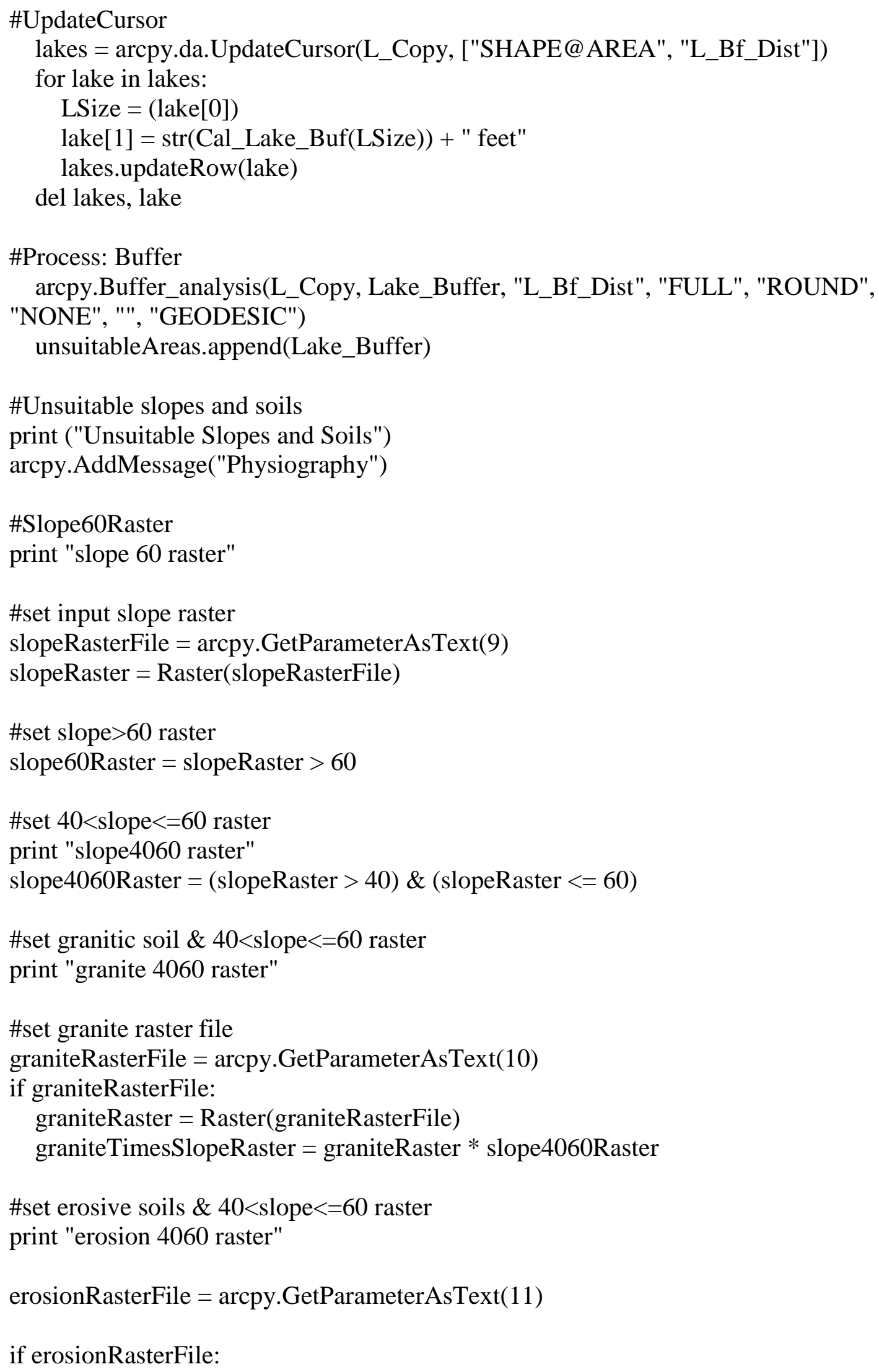




$$
\begin{aligned}
& \text { erosionRaster }=\text { Raster }(\text { erosionRasterFile }) \\
& \text { erosionTimesSlopeRaster }=\text { erosionRaster } * \text { slope4060Raster }
\end{aligned}
$$

\#set slopeErosionGraniteRaster

print "slopeErosoinGraniteRaster"

\#Handle no erosive soil or granitic soil data

if erosionRasterFile and graniteRasterFile:

slopeErosionGraniteRaster $=$ slope60Raster + erosionTimesSlopeRaster + graniteTimesSlopeRaster

elif erosionRasterFile:

slopeErosionGraniteRaster $=$ slope60Raster + erosionTimesSlopeRaster

elif graniteRasterFile:

slopeErosionGraniteRaster $=$ slope60Raster + graniteTimesSlopeRaster else:

slopeErosionGraniteRaster $=$ slope60Raster

\#SetNullValues

print "setnullvalues"

whereClause $=$ "VALUE $=0 "$

outRas $=$ SetNull( slopeErosionGraniteRaster, 1, whereClause $)$

\#create unsuitable physiography polygons

print "unsuitable physiography polygons"

inputraster $=$ outRas

slopesoilpolygon = "in_memoryllslopesoilpolygon"

arcpy.RasterToPolygon_conversion(inputraster, slopesoilpolygon, "SIMPLIFY",

"VALUE")

unsuitableAreas.append(slopesoilpolygon)

\#Merge Unsuitable Harvest Areas, Erase from Study Area print ("Removing Unsuitable Harvest Areas from Forest Boundary")

arcpy.AddMessage ("Removing Unsuitable Harvest Areas from Forest Boundary")

scratch $=$ arcpy.env.scratchGDB

Timber_Area_Boundary = scratch + "IITimber_Area_Boundary"

unsuitableMerge = "in_memoryllmerged_Features"

arcpy.Merge_management(unsuitableAreas, unsuitableMerge)

arcpy.Erase_analysis(forestBoundary, unsuitableMerge, Timber_Area_Boundary)

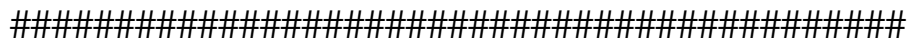


\#Network Analysis

print "Network Analysis"

arcpy.AddMessage ("Network Analysis")

print "Generating Fishnet"

arcpy.AddMessage ("Generating Fishnet")

\#Fishnet Cell Size

Cell_Size_Height $=$ arcpy.GetParameterAsText $(12)$

Cell_Size_Width $=$ arcpy.GetParameterAsText(13)

\#Fishnet Outputs

Output_Fishnet_Cells = "in_memoryl|OutputFishnetCells"

Output_Fishnet_Points = "in_memory $\backslash$ OutputFishnetCells_label"

Fishnet_Points_Clip = "in_memoryl\FishnetPointsClip"

\#Set the Coordinate System

dataset $=$ forestBoundary

desc $=$ arcpy.Describe(dataset)

spatialReference $=$ desc . spatialReference

arcpy.env.outputCoordinateSystem $=$ spatialReference

\# Process: Create Fishnet

print ("Create Fishnet")

fishnetExtent $=$ arcpy.Describe(Timber_Area_Boundary).extent

origin $=\operatorname{str}($ fishnetExtent.lowerLeft.X) $+" "+\operatorname{str}($ fishnetExtent.lowerLeft.Y)

yAxis $=\operatorname{str}($ fishnetExtent.lowerLeft.X) $+" ~ "+\operatorname{str}($ fishnetExtent.lowerLeft.Y + 10)

corner $=\operatorname{str}($ fishnetExtent.upperRight.X) $+"$ " + str(fishnetExtent.upperRight.Y)

arcpy.CreateFishnet_management(Output_Fishnet_Cells, origin, yAxis,

Cell_Size_Width, Cell_Size_Height, "", "", corner, "LABELS", fishnetExtent, "POLYGON")

\# Process: Clip Fishnet Cells to Study Area Boundary

print ("Clipping")

arcpy.Clip_analysis(Output_Fishnet_Points, Timber_Area_Boundary, Fishnet_Points_Clip, "")

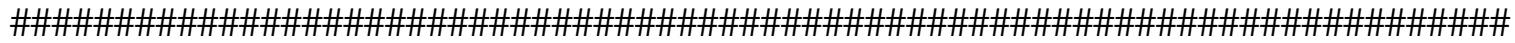

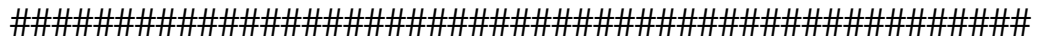

print ("Calculating Travel Times")

arcpy.AddMessage ("Calculating Travel Times")

Road_Network $=$ arcpy.GetParameterAsText(14) 


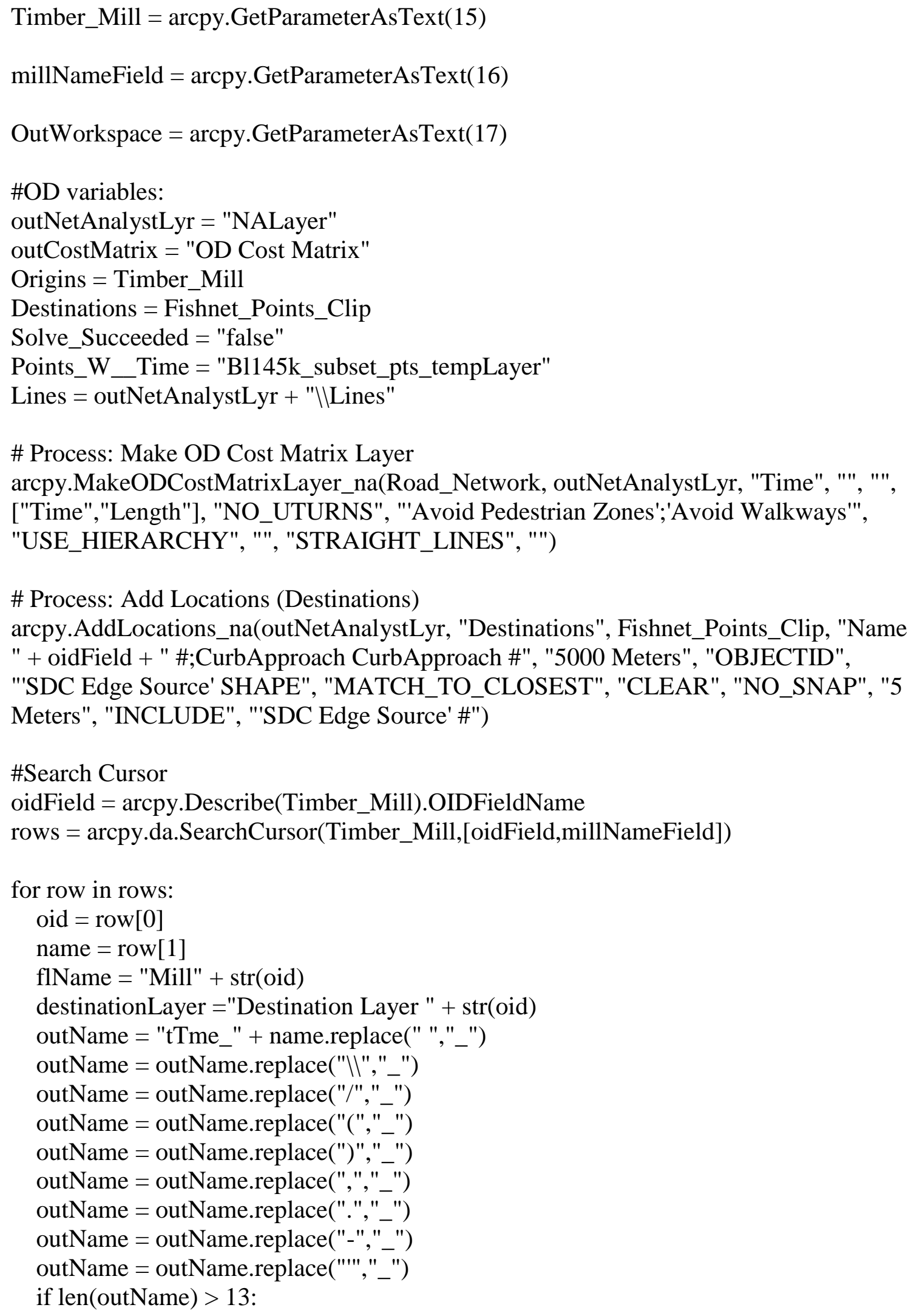


outName $=$ outName[0:13]

outFc = OutWorkspace + "II" + outName

print ("Executing OD for " + name)

arcpy.MakeFeatureLayer_management(Timber_Mill,flName, oidField + " = " + $\operatorname{str}($ oid))

\# Process: Add Locations (Origins)

arcpy.AddLocations_na(outNetAnalystLyr, "Origins", flName, "Name " +

millNameField + " \#", "5000 Meters", "", "'SDC Edge Source' SHAPE",

"MATCH_TO_CLOSEST", "CLEAR", "NO_SNAP", "5 Meters", "INCLUDE", "'SDC

Edge Source' \#")

\# Process: Solve

arcpy.Solve_na(outNetAnalystLyr, "SKIP", "CONTINUE", "")

print arcpy.GetMessages(1)

\# Process: Select Data

outLines $=$ arcpy.SelectData_management(outNetAnalystLyr, "Lines")

\#Join lines to points

print ("Add Join")

arcpy.MakeFeatureLayer_management(Destinations, destinationLayer)

arcpy.AddJoin_management(destinationLayer, "OBJECTID", Lines, "DestinationID",

"KEEP_ALL")

\# Process: Spatial Join

print ("Spatial Join")

arcpy.SpatialJoin_analysis(Timber_Area_Boundary, destinationLayer, outFc,

"JOIN_ONE_TO_ONE", "KEEP_ALL", "", "CLOSEST_GEODESIC", "", "")

\#Delete Unnecessary Fields

arcpy.DeleteField_management(outFc,["ODLines_Name"])

arcpy.DeleteField_management(outFc,["ODLines_ObjectID"])

arcpy.DeleteField_management(outFc,["ODLines_OriginID"])

arcpy.DeleteField_management(outFc,["ODLines_DestinationID"])

arcpy.DeleteField_management(outFc,["ODLines_DestinationRank"])

print ("Analysis for " + outName + " completed")

arcpy.AddMessage ("Analysis for " + outName + " completed")

\#Delete Timber Area Boundary

if arcpy.Exists(Timber_Area_Boundary):

arcpy.Delete_management(Timber_Area_Boundary)

print "Analysis Complete"

arcpy.AddMessage ("Analysis Complete") 